\title{
Novel chimpanzee adenovirus-vectored respiratory mucosal tuberculosis vaccine: overcoming local anti-human adenovirus immunity for potent TB protection
}

M Jeyanathan ${ }^{1,3}$, N Thanthrige-Don ${ }^{1,3}, \mathrm{~S}$ Afkhami ${ }^{1,3}, \mathrm{R} \mathrm{Lai}^{1}$, D Damjanovic ${ }^{1}$, A Zganiacz ${ }^{1}$ X Feng ${ }^{1}$,

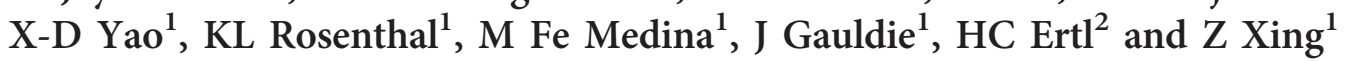

Pulmonary tuberculosis (TB) remains to be a major global health problem despite many decades of parenteral use of Bacillus Calmette-Guérin (BCG) vaccine. Developing safe and effective respiratory mucosal TB vaccines represents a unique challenge. Over the past decade or so, the human serotype 5 adenovirus (AdHu5)-based TB vaccine has emerged as one of the most promising candidates based on a plethora of preclinical and early clinical studies. However, anti-AdHu5 immunity widely present in the lung of humans poses a serious gap and limitation to its real-world applications. In this study we have developed a novel chimpanzee adenovirus 68 (AdCh68)-vectored TB vaccine amenable to the respiratory route of vaccination. We have evaluated AdCh68-based TB vaccine for its safety, T-cell immunogenicity, and protective efficacy in relevant animal models of human pulmonary TB with or without parenteral BCG priming. We have also compared AdCh68-based TB vaccine with its AdHu5 counterpart in both naive animals and those with preexisting anti-AdHu5 immunity in the lung. We provide compelling evidence that AdCh68-based TB vaccine is not only safe when delivered to the respiratory tract but, importantly, is also superior to its AdHu5 counterpart in induction of T-cell responses and immune protection, and limiting lung immunopathology in the presence of preexisting anti-AdHu5 immunity in the lung. Our findings thus suggest AdCh68-based TB vaccine to be an ideal candidate for respiratory mucosal immunization, endorsing its further clinical development in humans.

\section{INTRODUCTION}

Pulmonary tuberculosis (TB) caused by Mycobacterium tuberculosis (M.tb) has haunted mankind for thousands of years and still remains a top infectious killer. ${ }^{1-4} \mathrm{~TB}$ causes $\sim 1.4$ million deaths and 9 million new cases each year. An estimated one-third of the world population is latently infected by M.tb and $5-10 \%$ of these people develop active TB some time in their lives. Despite availability of antibiotics, increased multidrugresistant or extensively drug-resistant $\mathrm{TB}$ cases continue to pose a global threat. Bacillus Calmette-Guérin (BCG), which has been used in humans for many decades, remains the only licensed anti-TB vaccine. BCG is given once via the skin shortly after birth in most countries. Although BCG effectively protects against disseminated childhood TB, it has failed to effectively control adolescent and adult pulmonary TB. ${ }^{3,5}$ Thus, there is an urgent need to develop novel TB vaccines that can be used for effective boost vaccination following parenteral BCG priming in humans. ${ }^{6}$

One of the immune evasion strategies that $M . t b$ utilizes is to significantly slow down the appearance of T-cell immunity in the lung. ${ }^{7}$ Such delay or "immunological gap" allows M.tb a foothold in the lung before immunity is established, ${ }^{7-10}$ and this is likely one of the reasons for the ineffectiveness of parenteral BCG vaccination in humans. ${ }^{6,7}$ Therefore, it is believed that

\footnotetext{
${ }^{1}$ McMaster Immunology Research Centre, Department of Pathology and Molecular Medicine and Michael G. DeGroote Institute for Infectious Disease Research, McMaster University, Hamilton, Ontario, Canada and ²Department of Immunology, The Wistar Institute, Philadelphia, Pennsylvania, USA. Correspondence: Z Xing (xingz@mcmaster.ca) ${ }^{3}$ The first three authors contributed equally to this work.
} 
effective boost vaccination strategies ought to fill such a gap in T-cell immunity in the lung. ${ }^{6,7,11,12}$ Recent preclinical research from us and others has identified the respiratory mucosal route of vaccination to be the most effective way to equip the lung, particularly the surface of the respiratory mucosa (the airway luminal compartment), with protective T-cell immunity. ${ }^{6,11-14}$ Mucosal vaccination does so via activating mucosal-specialized dendritic cells and upregulating mucosa-homing molecules on $\mathrm{T}$ cells. ${ }^{10,15,16}$ Nevertheless, almost all of the TB vaccine candidates currently in the TB vaccine pipeline are still being developed as parenteral vaccines for human application. ${ }^{4}$ In this regard, the immune adjuvant used for parenteral delivery of protein-based TB vaccines is unsafe for respiratory mucosal applications. ${ }^{17,18}$ Replicating mycobacterial-based TB vaccines are known to cause granulomatous inflammation and cannot be easily cleared from the lung, thus deemed unsuitable for respiratory mucosal vaccination. Recent report on the inability of intradermally delivered MVA (modified vaccinia Ankara)based TB vaccine to enhance protective immunity in a major milestone phase $2 \mathrm{~b}$ trial $^{19}$ speaks further to the urgency and the importance of the effort in developing different vaccination strategies such as mucosal vaccination against pulmonary TB.

However, developing both safe and effective respiratory mucosal-deliverable vaccines represents a unique challenge and has recently been identified, at the National Institutes of Health (NIH)/Aeras Aerosol TB Vaccine Workshop, to be a new priority. ${ }^{6,13-15,20}$ Recombinant, replication-deficient virus-vectored $\mathrm{TB}$ vaccine platforms represent the most attractive approach for respiratory mucosal vaccination. ${ }^{12,21-23}$ Based on its unrivaled potency, the group $C$ human serotype 5 adenoviral vector system (AdHu5) has been widely used for developing vaccines against infectious diseases and cancer. ${ }^{21,22,24-26}$ Indeed, an AdHu5-based TB vaccine developed by us was robustly protective, particularly when given via the respiratory tract, in a number of animal models. ${ }^{6,12,21}$ This vaccine has also recently been evaluated following intramuscular administration in a phase 1 clinical study. ${ }^{27}$ However, a major gap and limitation to the application of AdHu5-based vaccine to humans is the pervasive preexisting anti-AdHu5 immunity resulting from respiratory exposure to $\mathrm{AdHu} 5$ virus that not only compromises the potency of the vaccine but may also pose a safety concern when given to HIV high-risk populations. ${ }^{22,24,25,28-32}$ Although human adenoviruses of rare serotypes such as AdHu35 may circumvent this issue, such vector is poorly immunogenic because of its strong type 1 interferon (IFN)-inducing ability. ${ }^{33-35}$

Recently, replication-defective chimpanzee-derived adenoviruses (AdCh) have become an attractive alternative to human adenoviral counterparts. ${ }^{33,35}$ Neutralizing antibodies against AdCh vectors are rarely found in humans. ${ }^{29,33-35}$ Furthermore, as most AdCh viruses utilize the same cell entry receptors as AdHu5, its immunogenicity is comparable to that by AdHu5. The large-scale production is also readily achievable as the same viral packaging cell line for AdHu 5 can be used for amplifying AdCh vectors. Thus, AdCh technology has recently been exploited to develop vaccines for malaria, HIV, Ebola, and hepatitis C virus. ${ }^{33,35-39}$ However, up to date, AdCh vector technology has not been translated to developing TB vaccines. Furthermore, its potential for respiratory mucosal application and the effect of pulmonary anti-AdHu5 immunity on its potency are still poorly understood.

In this study, for the first time we have applied the new technology for developing a novel replication-defective AdChbased TB vaccine for respiratory mucosal application. By using preclinical models of human pulmonary TB with or without preexisting anti-AdHu5 immunity, we provide compelling evidence that respiratory-mucosally delivered AdCh-based TB vaccine activates high levels of T-cell responses, provides robust protection, and limits lung pathology at a capacity comparable to or even better than its AdHu5 counterpart. Importantly, contrary to AdHu5Ag85A, respiratory mucosal AdCh68Ag85A-induced protective T-cell immunity is minimally affected by anti-AdHu5 immunity preexisting locally in the lung. Our study supports the AdCh-based TB vaccine to be an ideal candidate for respiratory mucosal immunization in humans and thus warrants its further clinical development.

\section{RESULTS}

\section{Molecular construction and characterization of a} chimpanzee adenovirus vector AdCh68-based TB vaccine A replication-deficient chimpanzee type 68 adenovirus was constructed to encode a secreted form of Ag85A, a highly immunogenic secreted antigen of M.tb (AdCh68Ag85A), by using a recently described direct cloning technology ${ }^{40}$ (Figure 1a). Expression of Ag85A is under control of a murine cytomegalovirus promoter and its secretion by mammalian cells is promoted by a human tissue plasminogen signal peptide sequence (tissue plasminogen activator). All Ag85A genetic expression elements in AdCh68Ag85A are identical to those in its human type 5 adenovirus-based TB vaccine AdHu5Ag85A. ${ }^{41}$ AdCh68Ag85A was packaged and propagated in 293 cells and purified under the Good Laboratory Practice conditions. Using a monoclonal antibody specific for Ag85A, the production and secretion of Ag85A protein were characterized in lung epithelial A549 cells by western immunoblotting. The expected size of $\sim 30 \mathrm{kDa}$ Ag85A protein was detected in both the culture supernatant and cellular lysate from A549 cells infected with AdCh68Ag85A but not from those infected with empty vector AdCh68 (Figure 1b). The levels of Ag85A antigen production by AdCh68Ag85A were found to be comparable to those from the cells infected with the same dose of AdHu5Ag85A (Figure 1b). These observations form the basis to compare AdCh68Ag85A with AdHu5Ag85A for their safety, immunogenicity, and protective efficacy in vivo.

\section{Respiratory mucosal inoculation with AdCh68Ag85A elicits desired innate immune activation and minimal tissue inflammation in the lung}

To begin evaluating the suitability of AdCh68Ag85A for respiratory mucosal vaccination, we first assessed to which degree the vector induced an inflammatory reaction in the lung. To this end, mice were inoculated intranasally (i.n.) with 
a
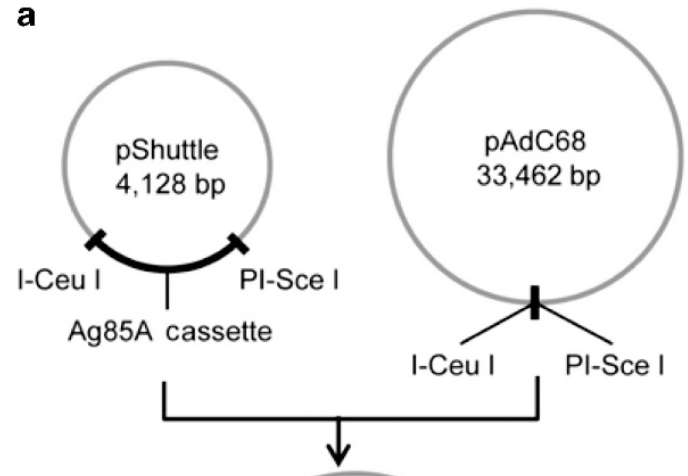

b

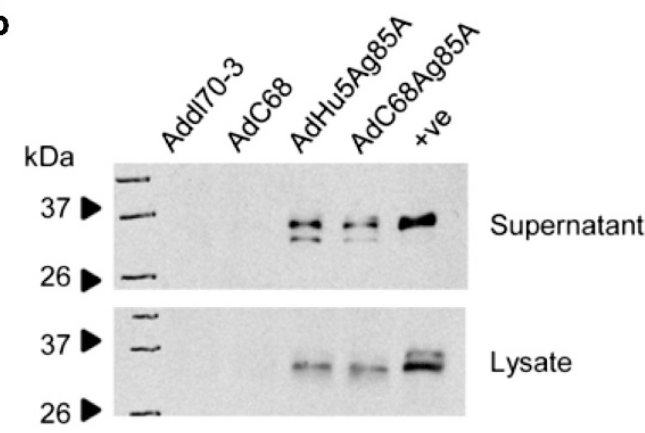

Figure 1 Molecular construction and characterization of AdCh68Ag85A. (a) An Ag85A genetic cassette was cloned into the pShuttle plasmid and was then excised with the indicated enzymes. The $\mathrm{I}$-Ceu1 and PI-Sce1-excised insert was subcloned into the I-Ceu1 and PI-Sce1 site of pAdC68 genomic clone to generate recombinant AdCh68Ag85A virus. (b) Characterization of Ag85A protein expression levels by AdCh68Ag85A-infected A549 cells was performed in culture supernatant and cellular lysate by using western blots and compared with the cells infected with AdHu5Ag85A. The empty viral vectors Addl70-3 and AdC68 were used as negative controls for $\mathrm{AdHu} 5 \mathrm{Ag} 85 \mathrm{~A}$ and AdCh68Ag85A, respectively. Recombinant Ag85A protein was used as a positive control (+ve). MCMV, murine cytomegalovirus promoter, tPA tissue plasminogen activator.

AdCh68Ag85A. As a comparison, a group of mice were inoculated with an equal dose of AdHu5Ag85A. By histopathological examination at days 1 and 3 after AdCh68Ag85A, only mild inflammatory infiltrates were seen in the peripheral bronchial and vascular areas compared with naive mice (Figure 2a). To further examine the inflammatory and innate immune responses in the lung, we analyzed the major cellular components in bronchoalveolar lavage fluid (BAL). The majority of innate immune cells in the BAL of AdCh68Ag85A lungs were alveolar macrophages at days 1 and 3 (Figure 2b). A degree of transient neutrophilic responses was observed only at day 1 but hardly at day 3 (Figure 2b). We also examined the responses of innate immune cytokines tumor necrosis factor- $\alpha$ and IFN $\beta$ in the BAL. Tumor necrosis factor- $\alpha$ is a type 1 immune cytokine, ${ }^{7}$ whereas IFN $\beta$ is a type I interferon usually induced during viral infection and has been shown to suppress anti-TB T helper type 1 cell immunity. ${ }^{42-44}$ Although intranasal inoculation of AdCh68Ag85A triggered a level of tumor necrosis factor- $\alpha$ production, the level of IFN $\beta$ was very low at the both time points (Figure 2c,d), in agreement with our previous finding. ${ }^{42}$

Upon comparison we found that the proinflammatory responses induced by AdCh68Ag85 were largely comparable to its human AdHu5Ag85A counterpart in histopathology (Figure 2a), inflammatory cellular responses (Figure 2b), and innate immune cytokine responses (Figure $\mathbf{2 c}, \mathbf{d}$ ). The above data together suggest that although AdCh68Ag85A possesses desired immune adjuvant properties, it is safe for respiratory mucosal administration. Furthermore, like its AdHu5 counterpart, AdCh68Ag85A triggers a negligible level of undesired type 1 IFN responses.

\section{Respiratory mucosal AdCh68Ag85A immunization induces robust $\mathrm{T}$-cell responses in the lung}

We next examined the T-cell immunogenicity of AdCh68Ag85A following respiratory mucosal vaccination. The mice were vaccinated i.n. once with AdCh68Ag85A as described above. For comparison, some mice were immunized i.n. with an equal dose of AdHu5Ag85A. T-cell responses were analyzed in the BAL and lung tissue at 2 and 4 weeks after vaccination (Figure 3a) by Ag85A tetramer (tet + ) and intracellular IFN $\gamma$ $(\mathrm{IFN} \gamma+)$ immunostaining. AdCh68Ag85A vaccination induced high levels of Ag85A tetramer-positive CD8 T cells (tet $+\mathrm{CD} 8+)$ in the airway lumen $(\mathrm{BAL})$ and lung interstitium (Lung) at 2 weeks (Figure 3b). By 4 weeks, the magnitude of tet + CD8 $\mathrm{T}$ cells contracted significantly. AdCh68Ag85A vaccination induced similarly increased responses of IFN $\gamma$ producing CD8 T cells in both the BAL and lung upon stimulation with an immunodominant Ag85A peptide (Figure 3c). AdCh68Ag85A vaccination also increased CD4 T-cell responses but at much lower levels than CD8 T-cell responses. In comparison, although AdHu5Ag85A vaccination also induced potent CD8 T-cell responses in the BAL and lung, the overall levels of T-cell responses at 2 weeks in the BAL were significantly lower than AdCh68Ag85A. Similar to AdCh68Ag85A, T-cell responses contracted significantly at 4 weeks after AdHu5Ag85A vaccination (Figure $3 \mathbf{b}, \mathbf{c}$ ).

To further evaluate the functionality of respiratory mucosal CD8 T cells activated by intranasal AdCh68Ag85A vaccination, we examined the cytolytic capability of these cells by using an in vivo intratracheal cytotoxic $\mathrm{T}$ cell (CTL) killing assay previously developed by us. ${ }^{45}$ Consistent with robust CD8 
a

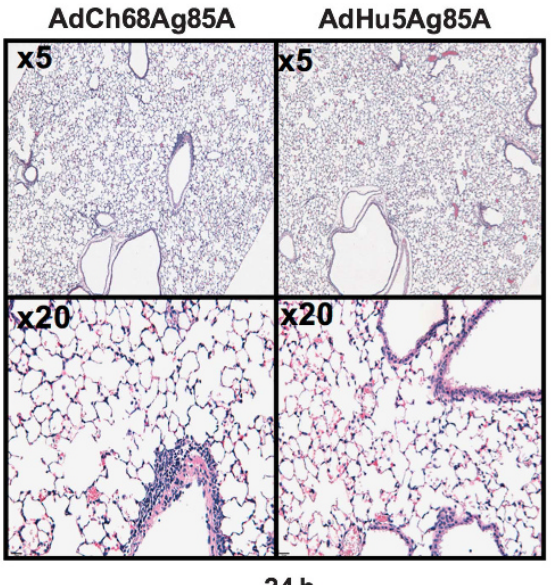

$24 \mathrm{~h}$

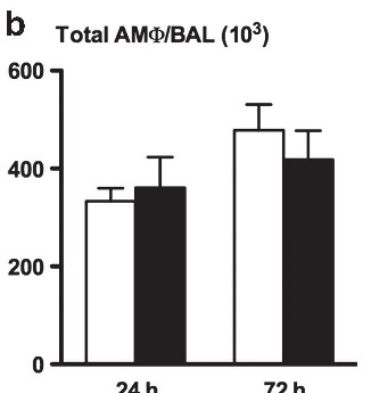

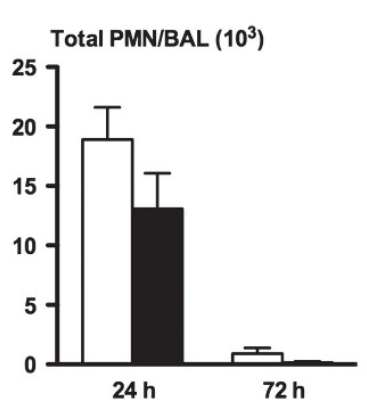
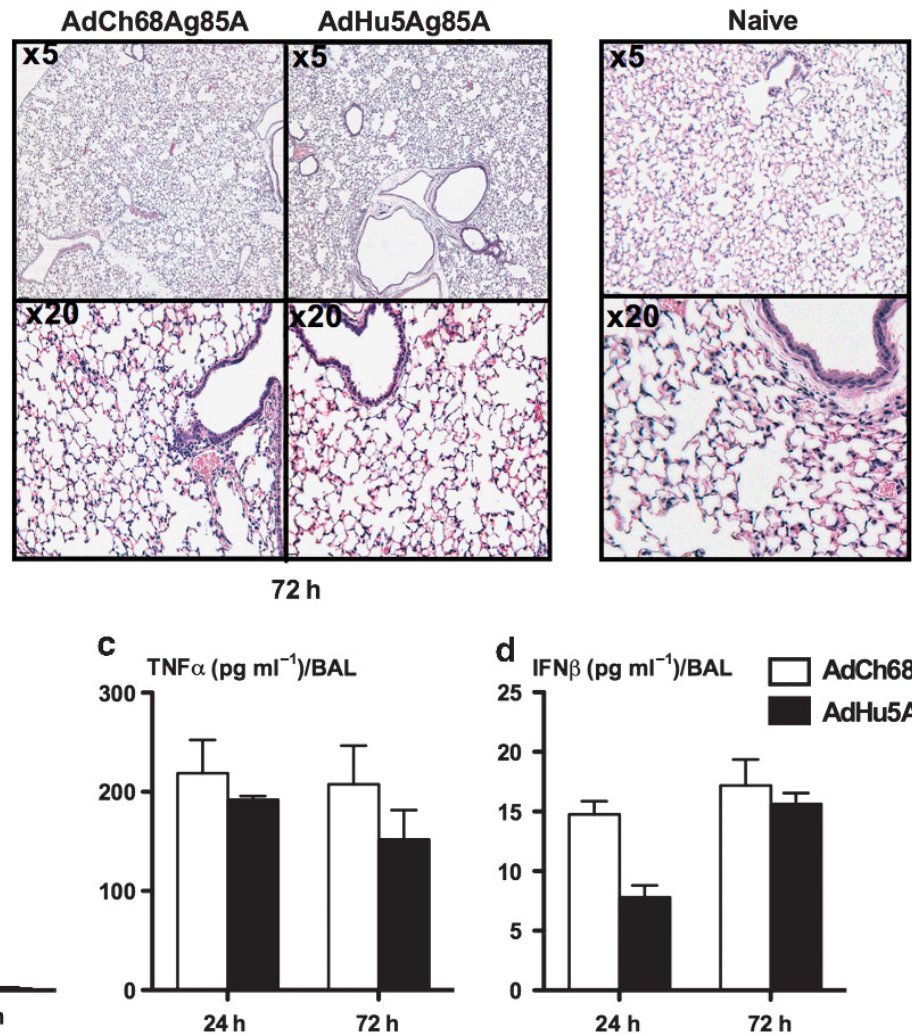

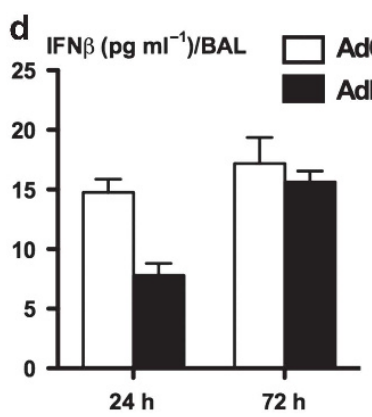

Figure 2 Innate immune activation and minimal lung inflammation by intranasal inoculation of AdCh68Ag85A. Mice were inoculated via intranasal route with $1 \times 10^{7}$ plaque-forming units (PFUs) AdCh68Ag85A or AdHu5Ag85A or left unimmunized (naive). Lungs were harvested at 24 or $72 \mathrm{~h}$ after inoculation and processed for histopathologic examination. (a) Light hematoxylin and eosin (H\&E) micrographs of original magnification $\times 5$ and $\times 20$ of lung sections are shown, representative of three mice per group per time point. (b) In separate experiments, the lungs were subject to bronchoalveolar lavage (BAL) and absolute numbers of alveolar macrophages (AMФs) and polymorphonuclear neutrophils (PMNs) in BAL were analyzed. The concentrations of (c) tumor necrosis factor- $\alpha(T N F \alpha)$ and (d) interferon- $\beta$ (IFN $\beta$ ) in BAL were determined by enzyme linked immunosorbent assay (ELISA). Data in $\mathbf{b}, \mathbf{c}, \mathbf{d}$ are expressed as the mean \pm s.e.m. of three to four mice per group per time point.

T-cell responses within the airway lumen by AdCh68Ag85A vaccination (Figure $3 \mathbf{b}, \mathbf{c}$ ), $>40 \%$ of CTL target killing was observed in the BAL at 2 weeks and the level of CTL declined by 4 weeks following vaccination (Figure 3d). In comparison, the level of CTL activity by intranasal AdHu5Ag85A was significantly lower at 2 weeks, but not at 4 weeks, than that by AdCh68Ag85A (Figure 3d). These data suggest that respiratory mucosal vaccination with AdCh68Ag85A is capable of inducing robust $\mathrm{T}$-cell responses on the respiratory mucosal surface and in the lung that are overall stronger than AdHu5Ag85A vaccination.

\section{Respiratory mucosal AdCh68Ag85A immunization activates CD8 $T$ cells of broadened epitope specificity}

As the chimpanzee adenovirus represents a novel viral vector utilized as a $\mathrm{TB}$ vaccine platform, we investigated whether AdCh68Ag85A vaccination would activate the T cells reactive to the same immunodominant and subdominant epitopes as AdHu5Ag85A vaccination. To this end, naive mice were vaccinated i.n. with AdCh68Ag85A or AdHu5Ag85A. At 2 weeks, lung mononuclear cells were analyzed by intracellular cytokine staining for IFN $\gamma$-producing CD8 T cells in response to a single peptide pool (p1-57) or 6 individual peptide pools (p1-10, p11-20, p21-30, p31-40, p41-50, and p51-57) of Ag85A (Figure 4a). Consistent with the data in Figure 3, although both AdCh68Ag85A and AdHu5Ag85A triggered high frequencies of antigen-specific CD8 $\mathrm{T}$ cells in response to single p1-57 peptide pool, the levels of such responses were higher by AdCh68Ag85A (Figure 4a). Significant T-cell responses were also seen with stimulation by individual peptide pools of p1120, p21-30, and p41-50 following AdCh68Ag85A and AdHu5Ag85A vaccination (the p11-20 pool contains the well-characterized immunodominant $\mathrm{H}-2^{\mathrm{d}}$ Ag85A CD8 T-cell epitope MPVGGQSSF or peptide 14/Ep-1). However, AdCh68Ag85A triggered noticeably much greater T-cell responses to p21-30 stimulation than AdHu5Ag85A (Figure 4a). These data suggest the emergence of an additional immunodominant Ag85A CD8 T-cell epitope, resulting from respiratory mucosal AdCh68Ag85A vaccination. We have also examined the effects of these two vaccines in $\mathrm{H}-2^{\mathrm{b}} \mathrm{C} 57 \mathrm{BL} / 6$ mice. At 2 weeks following intranasal vaccination, lung mononuclear cells were analyzed by intracellular cytokine staining for IFN $\gamma$-producing CD8 and CD4 $\mathrm{T}$ cells in response to 6 individual peptide pools of Ag85A described as above. 
a

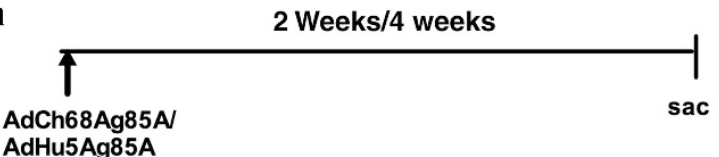

AdHu5Ag85A

b

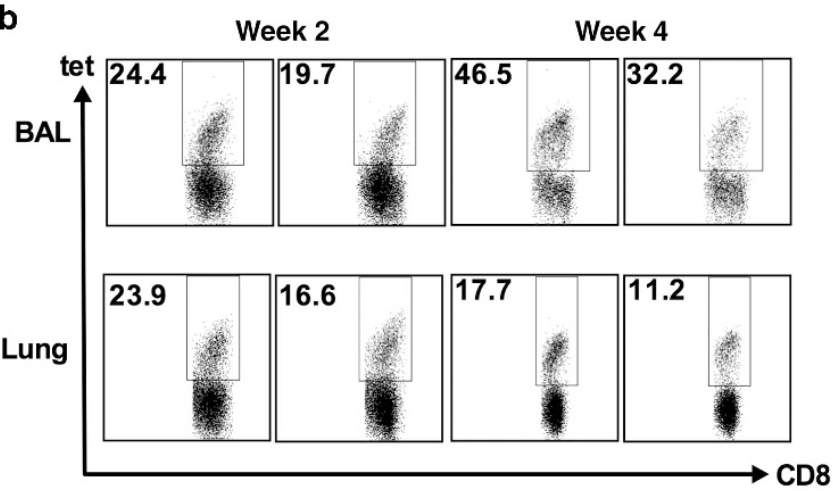

tet+CD8+ cells $\left(\times 10^{3}\right)$
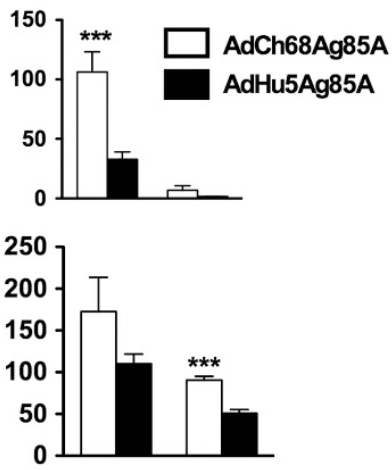

C

IFN- $\gamma+$ CD $8+$ cells $\left(\times 10^{3}\right)$
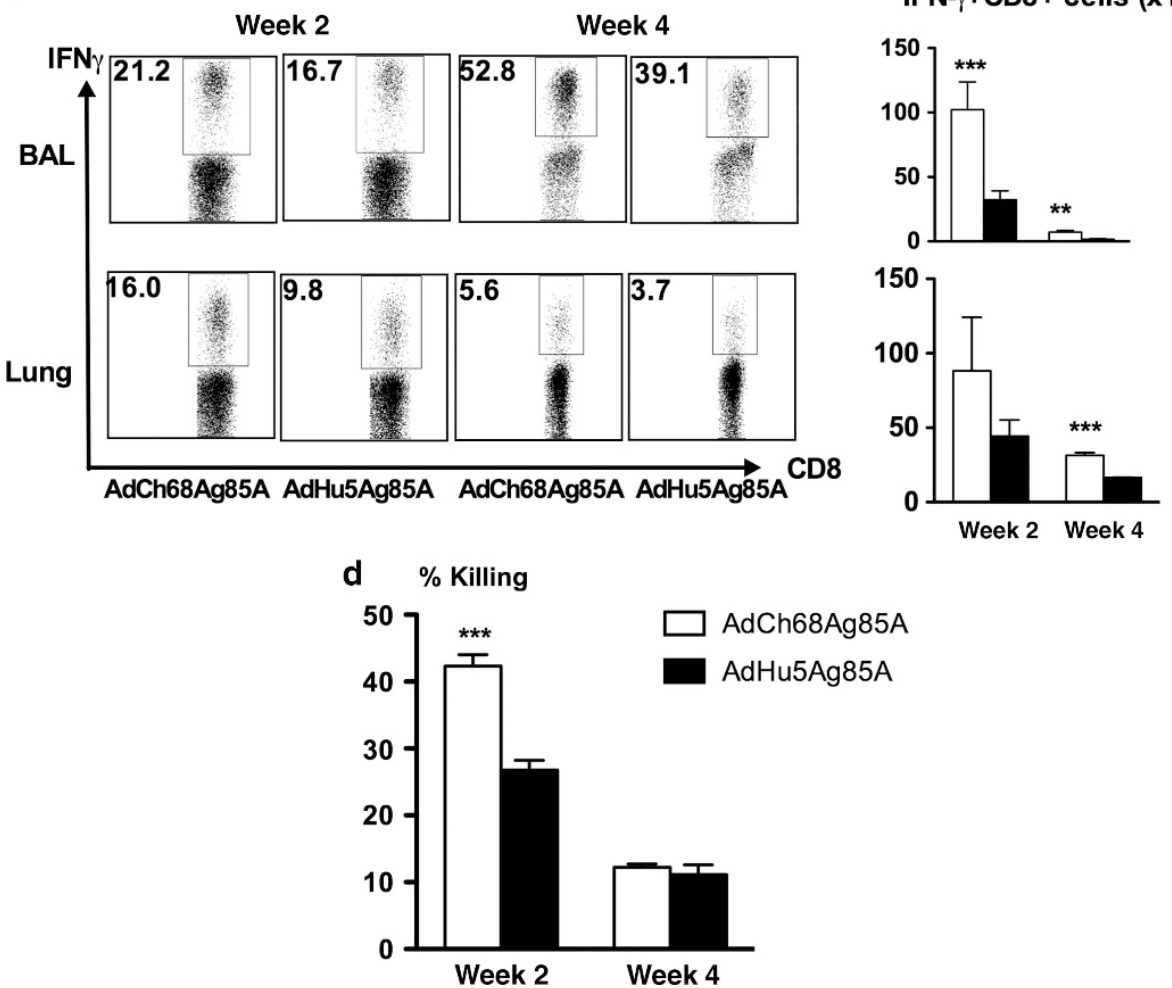

Figure 3 Robust T-cell responses in the lung by respiratory mucosal AdCh68Ag85A immunization. (a) Experimental schema. Intranasal immunization with $1 \times 10^{7}$ plaque-forming units (PFUs) AdCh68Ag85A or AdHu5Ag85A was carried out and animals were killed at weeks 2 and 4 after immunization. (b) Ag85A tetramer-specific CD8 T-cell responses in the airway lumen (bronchoalveolar lavage (BAL)) and the lung interstitium (Lung). Representative dotplots depicting frequencies of tetramer + CD8 T cells in the BAL and lung at week 2 and week 4 are shown. Bar graphs show absolute numbers of tetramer + CD8 T cells per BAL and per lung at week 2 and week 4 after immunization. (c) Ag85A-specific interferon- $\gamma$ (IFN $\gamma$ )-producing CD8 T-cell responses in the BAL and lung. Representative dotplots depicting frequencies of IFN $\gamma+$ CD8 T cells in the BAL and lung at week 2 and week 4 are shown. Bar graphs show absolute numbers of IFN $\gamma+$ CD8 T cells per BAL and per lung at week 2 and week 4 after immunization. (d) Cytotoxic T-cell (CTL) killing activity in the airway lumen. In vivo intratracheal CTL assay was performed at week 2 and week 4 after immunization. Bar graphs show percent in vivo killing of carboxyfluorescein succinimidyl ester (CFSE)-labeled CTL target cells. Data in $\mathbf{b}, \mathbf{c}, \mathbf{d}$ are expressed as the mean $\pm \mathrm{s}$.e.m. of three to four mice per group per time point. Data in $\mathbf{b}, \mathbf{c}$ are representative of three independent experiments. ${ }^{\star \star} P<0.01 ;{ }^{\star \star \star} P<0.001$ compared with AdHu5Ag85A.

Consistent with previous findings ${ }^{46}$ we found no major histocompatibility complex class I (MHC I)-restricted Ag85Aspecific CD8 T-cell responses in B6 hosts following either
AdHu5 or AdCh68 vaccination (data not shown). However, there were very small frequencies of MHC II-restricted CD4 T-cell responses detected that were comparable between 


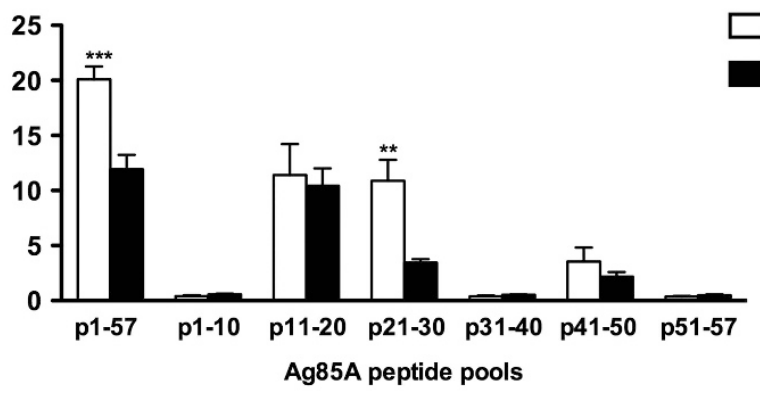

b

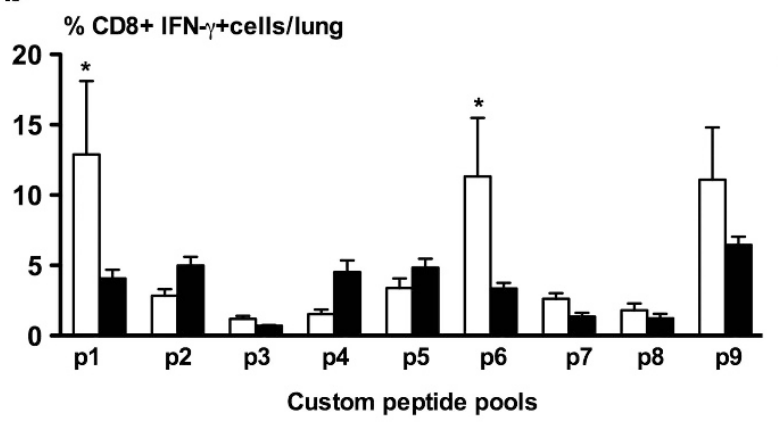

Matrix

\begin{tabular}{|l|l|}
\hline & \multicolumn{1}{|c|}{ Peptides } \\
\hline p1 & $21,22,23,24,25,26,27,28, \underline{\mathbf{2 9}}$ \\
p2 & $30,41,42,43,44,45,46,47,48$ \\
p3 & 49,50 \\
p4 & $21,24,27,30,43,46,49$ \\
p5 & $22,25,28,41,44,47,50$ \\
p6 & $23,26, \mathbf{2 9}, 42,45,48$ \\
p7 & $21,22,23,30,41,42,49,50$ \\
p8 & $24,25,26,43,44,45$ \\
p9 & $27,28, \underline{\mathbf{2 9}}, 46,47,48$ \\
\hline
\end{tabular}

C

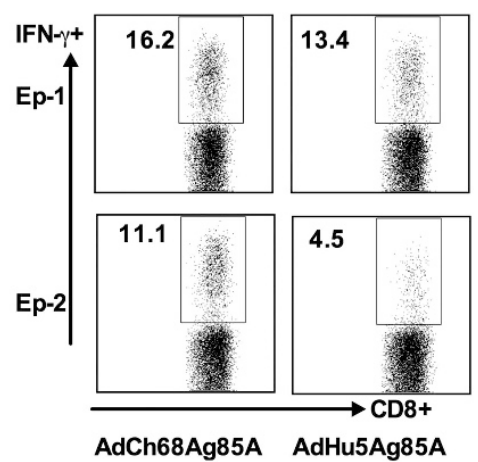

CD8+ IFN- - cells $\left(\times 10^{3}\right) / l u n g$

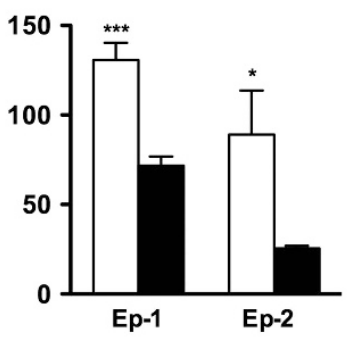

Figure 4 Activation of CD8 T cells of broadened epitope specificity by respiratory mucosal AdCh68Ag85A immunization. Mice were immunized via intranasal route with $1 \times 10^{7}$ plaque-forming units (PFUs) AdCh68Ag85A or AdHu5Ag85A. Lungs were harvested at week 2 after immunization and CD8 T-cell responses to Ag85A peptide pools or peptide were determined by interferon- $\gamma$ (IFN $\gamma$ ) intracellular cytokine staining (ICS). (a) Bar graph shows frequencies of CD8 + IFN $\gamma+$ cells in the lung responding to seven peptide pools of Ag85A. (b) Bar graph shows frequencies of CD8 + IFN $\gamma+$ cells in the lung responding to newly designed nine peptide pools. These new peptide pools were designed in such a way that each peptide is present only in three unique pools, according to the Matrix shown in the table. The newly emerged immunodominant peptide 29 is highlighted. (c) Representative dotplots show frequencies of CD8 + IFN $\gamma+$ cells in the lung responding to a conventional immunodominant Ag85A peptide Ep-1 and to a newly emerged immunodominant Ag85A peptide Ep-2. Bar graph shows absolute numbers of CD8 + IFN $\gamma+$ cells in the lung responding to Ep-1 and Ep-2 peptides. Data in $\mathbf{a}, \mathbf{b}, \mathbf{c}$ are expressed as the mean \pm s.e.m. of three to four mice per group, representative of two independent experiments. ${ }^{\star} P<0.05$; ${ }^{\star \star} P<0.01$; ${ }^{\star * \star} P<0.001$ compared with AdHu5Ag85A.

AdHu5 and AdCh68 vaccinations (Supplementary Table S1 online). The level of such MHC II-restricted responses in B6 mice was comparable to that detected in $\mathrm{BALB} / \mathrm{c}$ mice (data not shown). These data together suggest that $\mathrm{AdHu} 5$ and AdCh68 vaccinations predominantly generate high levels of MHC I-restricted CD8 T-cell responses in BALB/c, but not in C57BL/6, hosts.

To further identify the potential additional CD8 T-cell epitopes present in the p21-30 and p41-p50 pools in BALB/c mice, a new set of 9 peptide pools with the peptides p21-p30 and p41-p50 were developed with each pool containing 2-9 peptides according to a previously described peptide-mapping matrix $^{47}$ (Figure $\mathbf{4 b}$, Matrix). In so doing, each peptide is present only in three unique pools. Significantly raised T-cell responses against pools $\mathrm{p} 1, \mathrm{p} 6$, and $\mathrm{p} 9$ were identified only in AdCh68Ag85A-vaccinated, but not AdHu5Ag85A-vaccinated, animals (Figure $4 \mathbf{b}$ ). Thus, the likely new candidate epitope was peptide 29, a 15-mer peptide highlighted in Figure 4b, Matrix table. To further verify it, by using an Immune Epitope Database (http://tools.immuneepitope.org/) we identified the highest probable CD8 T-cell epitope sequence in peptide 29 to be VYAGAMSGL (Ep-2). Using its synthesized 9-mer peptide, we confirmed its immunodominance by demonstrating a robust CD8 T-cell response induced only by intranasal 

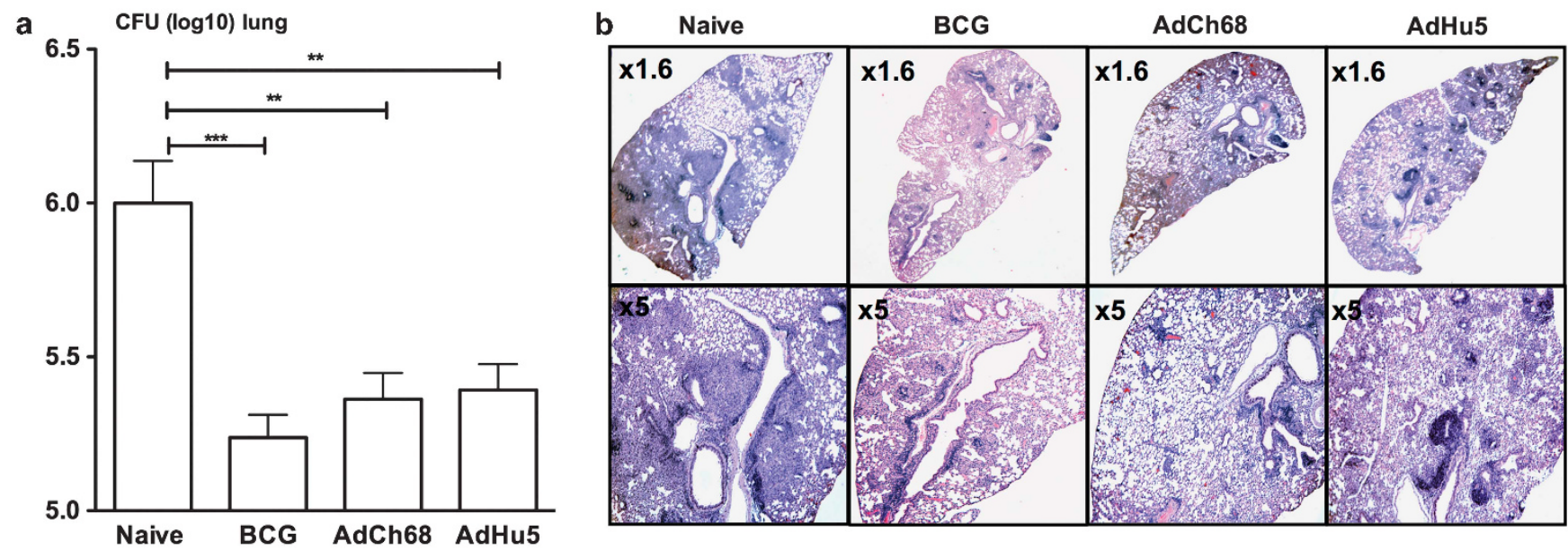

Figure 5 Potent protection against pulmonary tuberculosis (TB) by respiratory mucosal AdCh68Ag85A immunization in naive hosts. (a) Naive mice were left unimmunized (naive) or immunized subcutaneously with Bacillus Calmette-Guérin; BCG), intranasally with $1 \times 10^{7}$ plaque-forming units (PFUs) AdCh68Ag85A (AdCh68) or with AdHu5Ag85A (AdHu5). At 4 weeks after immunization, mice were challenged with Mycobacterium tuberculosis (M.tb) and the lungs were harvested for analysis 4 weeks after infection. (a) Bar graph shows the colony-forming units (CFUs) of M.tb in the lung. (b) Representative histopathologic images from hematoxylin and eosin (H\&E)-stained lung sections of three mice per group are shown at original magnification $\times 1.6$ and $\times 5$. Data in a are expressed as the mean \pm s.e.m. of four to five mice per group, representative of two independent experiments. ${ }^{\star \star} P<0.01 ;{ }^{\star \star \star} P<0.001$.

AdCh68Ag85A, but not AdHu5Ag85A, vaccination (Figure 4c). In comparison, the $\mathrm{CD} 8 \mathrm{~T}$ cells activated by both AdCh68Ag85A and AdHu5Ag85A vaccination well responded to stimulation by the common immunodominant peptide MPVGGQSSF (Ep-1) (Figure 4c), although as shown in Figure 3c, AdCh68Ag85A triggered higher levels of responses. These data indicate that compared with AdHu5Ag85A, mucosal AdCh68Ag85A vaccination is able to activate CD8 T cells of broader epitope specificity in $\mathrm{H}-2^{\mathrm{d}}$ Balb/c hosts.

\section{Respiratory mucosal AdCh68Ag85A immunization provides robust protection against pulmonary tuberculosis in naive hosts}

Having demonstrated the potent T-cell immunogenicity of AdCh68Ag85A, we next assessed its protective efficacy against pulmonary $\mathrm{TB}$ in naive animals. To this end, naive mice were vaccinated as above with AdCh68Ag85A (AdCh68) or AdHu5Ag85A (AdHu5). As a comparison, some mice were subcutaneously (s.c.) vaccinated with BCG (BCG) or were not vaccinated at all (naive). At 4 weeks after immunization, mice were challenged via the airway with virulent M.tbH37Rv. Protective efficacy in the lung was evaluated at 4 weeks after challenge. Indeed, respiratory mucosal AdCh68 vaccination markedly reduced the levels of M.tb infection in the lung compared with unvaccinated animals (Figure 5a). The magnitude of enhanced protection by AdCh68 was comparable to that by AdHu5 counterpart or by parenteral BCG vaccination (Figure 5a).

As the relative amount of M.tb-associated lung immunopathology is an even more sensitive and reliable indicator of protection in some other animal models than in murine models of $\mathrm{TB}$, we examined and compared lung immunopathology. In keeping with significantly improved protection by respiratory mucosal AdCh68 vaccination, there was markedly reduced overall granulomatous inflammation and tissue injury in the lung of AdCh68-vaccinated animals, contrasting widespread pronounced granulomatous lesions in the lung of unvaccinated animals (Figure 5b). Of note, the lung of AdCh68 animals had less immunopathology than the lung of AdHu5vaccinated animals (Figure 5b). Thus, compared with AdHu5 lungs, the sizes of granulomatous lesion were smaller and there was less bronchial injury in the lung of AdCh68-vaccinated animals. These data together suggest that compared with its AdHu5 counterpart, respiratory mucosal AdCh68Ag85A immunization provides overall further improved protective immunity against pulmonary TB in the lung of naive animals.

Different from its AdHu5 counterpart, respiratory mucosal AdCh68Ag85A immunization is able to provide potent antituberculosis immunity in hosts with preexisting antiAdHu5 immunity in the lung

AdCh vectors have recently emerged to be an attractive alternative to AdHu5 counterparts for the development of human vaccines because of prevalence of anti-AdHu5 immunity in humans. ${ }^{22,24,33,35}$ Indeed, it has been widely accepted that anti-AdHu5 antibodies do not crossreact with AdCh. ${ }^{22,24}$ However, it remains largely unclear whether anti-AdHu5 $\mathrm{T}$ cells may negatively affect the potency of AdCh-based vaccine. ${ }^{30}$ Of importance, little is understood about whether AdCh vector-based vaccine would remain effective when delivered via the respiratory tract-the natural route of human AdHu5 infection-in the context of local anti-AdHu5 immunity. This is an important issue to address as Ad-based $\mathrm{TB}$ vaccines were developed primarily as a novel platform for respiratory mucosal delivery in humans. We began to address this issue first by examining to which extent the preexisting 
anti-AdHu5 immunity would negatively affect the $\mathrm{T}$ cellactivating potency of parenteral (intramuscular) vaccination with AdC68Ag85A or AdHu5Ag85A. Thus, naive mice were first exposed to wild-type AdHu5 virus (WtAd) via the intranasal route (exposed). Such respiratory WtAd exposure led to significant levels of anti-AdHu5 immunity as exemplified by markedly elevated total anti-AdHu 5 IgG and IgA antibodies and raised levels of AdHu5-neutralizing antibody titers both locally in the lung (BAL) and systemically in the peripheral blood (serum) (Supplementary Figure S1). At 4 weeks after WtAd infection, mice were vaccinated intramuscularly with AdCh68Ag85A (AdCh68) or AdHu5Ag85A (AdHu5) (Figure 6a). The control animals were vaccinated similarly without WtAd exposure (unexposed). Ag85A-specific T-cell responses were examined in the BAL, lung, and spleen. As expected, ${ }^{6,11,12}$ intramuscular AdCh68 or AdHu5 vaccination of unexposed animals failed to elicit any airway luminal $\mathrm{T}$ cells (BAL), whereas WtAd-exposed animals had similarly increased BAL $\mathrm{T}$ cells following intramuscular AdCh68 or AdHu5 vaccination (Figure 6b). On the other hand, compared with unexposed animals, significantly increased numbers of CD8 T cells were observed in the lung of WtAd-exposed intramuscular AdC68 animals (Figure 6b). On the contrary, significantly reduced numbers of CD8 T cells were observed in the lung of WtAd-exposed intramuscular AdHu animals (Figure 6b). Similarly, reduced CD8 T cells were seen in the spleen of only WtAd-exposed AdHu5-vaccinated animals, but not WtAdexposed AdCh68 vaccinated animals (Figure 6b). These data together support and extend the previous findings that preexisting circulating anti-AdHu5 immunity dampens the potency of parenterally administered AdHu5 gene transfer vectors but not those based on chimpanzee adenovirus. ${ }^{24,48}$

After demonstrating that different from AdHu5Ag85A vaccination, intramuscular AdCh68Ag85A vaccination can indeed bypass the preexisting anti-AdHu 5 immunity to induce potent $\mathrm{T}$-cell responses, we next investigated to which extent this could be reproduced upon respiratory mucosal AdCh68 vaccination in WtAd-exposed animals. To this end, mice were exposed to WtAd via the intranasal route and at 4 weeks after WtAd infection, they were vaccinated i.n. with AdCh68 or AdHu5 vaccine (Figure 6c). The (WtAd) unexposed naive mice were vaccinated i.n. as controls. T-cell responses were subsequently examined in the BAL and lung. Of interest, compared with unexposed animals, preexisting anti-AdHu5 immunity in the respiratory mucosa appeared to result in reduced airway luminal (BAL) T-cell responses following either respiratory mucosal AdCh68 or AdHu5 vaccination (Figure 6d). However, preexisting anti-AdHu5 immunity had little negative effects on $\mathrm{T}$ cells in the lung interstitium of AdCh68-vaccinated animals, whereas it significantly reduced T-cell responses in the lung of AdHu5-vaccinated animals (Figure 6d). Overall, intranasal AdCh68Ag85A vaccination triggered much greater antigen-specific $\mathrm{T}$-cell responses in the entire lung (both airway luminal and lung interstitial compartments) than intranasal AdHu5Ag85A vaccination in respiratory WtAd-exposed animals.
To investigate whether the greater T-cell responses seen in the lung of WtAd-exposed, AdCh68-vaccinated animals would lead to better immune protection from pulmonary TB, groups of mice were exposed i.n. to WtAd and subsequently vaccinated via the respiratory mucosal route with AdCh68 or AdHu5 vaccine as above and then challenged via the airway with virulent M.tbH37Rv (Figure 6e). Relative levels of protective efficacy in the lung were assessed at 4 weeks after M.tb infection. Consistent with the protection data shown in Figure 5a, (WtAd) unexposed animals were similarly protected in the lung by intranasal AdCh68 or AdHu5 vaccination (Figure 6f). Also, consistent with the immunogenicity data (Figure 6d), preexisting anti-AdHu5 immunity had little negative effects on immune protection of AdCh68-vaccinated animals, whereas it significantly reduced protection, leading to markedly increased M.tb infection in the lung of AdHu5-vaccinated animals (Figure 6f). As the above set-up did not directly address whether preexposure to WtAd on its own had an effect on immune protection, in a separate experiment mice were exposed to WtAd and at 4 weeks after exposure mice were left either unvaccinated or vaccinated with AdHu5Ag85A or AdCh68Ag85A. At 4 weeks after vaccination, mice were challenged with M.tb and bacterial burden in the lung was assayed. As expected, compared with WtAd-unexposed and unvaccinated mice (naive/unexposed), WtAd exposure alone without vaccination (naive/exposed) had little significant enhancing effects on protection against M.tb infection (Figure 6g). Importantly, compared with naive/exposed hosts, AdHu5Ag85A vaccination in the presence of antiAdHu5 immunity (AdHu5/exposed) failed to provide significantly enhanced protection, whereas AdCh68Ag85A vaccination in the presence of anti-Ad5 immunity (AdCh68/exposed) remained robustly protective (Figure $\mathbf{6 g}$ ). The above data together suggest that respiratory mucosal AdCh68Ag85A immunization remains capable of triggering potent protective $\mathrm{T}$-cell immunity against pulmonary TB in the animals with preexisting anti-AdHu5 immunity in the lung. In contrast, the potency of respiratory mucosal immunization with its human counterpart AdHu5Ag85A vaccine is markedly reduced by such local anti-AdHu5 immunity.

\section{Respiratory mucosal AdCh68Ag85A immunization boosts protective T-cell immunity against pulmonary tuberculosis in parenteral BCG-primed hosts}

Like a number of other candidate $\mathrm{TB}$ vaccines that are currently in the clinical development, AdCh68Ag85A was developed for boost vaccination of parenteral BCG-primed humans. Having systematically investigated the immunogenicity and protective efficacy of respiratory mucosal AdCh68Ag85A vaccination described above, we next set out to examine its effectiveness in boosting protective immunity following parenteral BCG priming. We first examined its effect on T-cell responses. To this end, naive mice were primed s.c. with BCG. At 4 weeks after BCG priming, mice were boosted i.n. with AdCh68Ag85A (BCG/AdCh68) (Figure 7a). Control mice received only BCG (BCG) or AdCh (AdCh) vaccination. At 4 weeks after boosting, 
a

Unexposed/exposed to WtAd (intranasal)

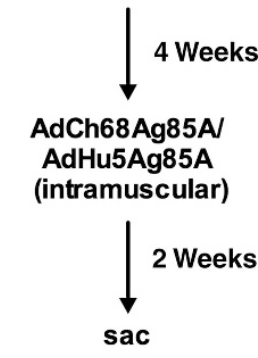

c

Unexposed/exposed to WtAd (intranasal)<smiles>C[13CH3]</smiles>

AdCh68Ag85A

AdHu5Ag85A

(intranasal)

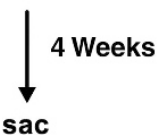

e

Unexposed/exposed to WtAd (intranasal)

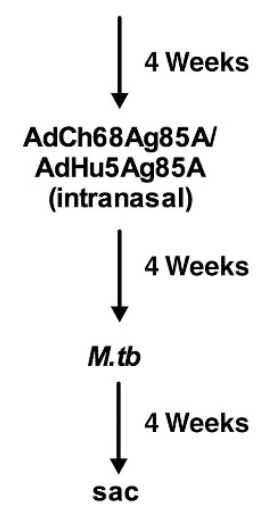

b

BAL

Lung
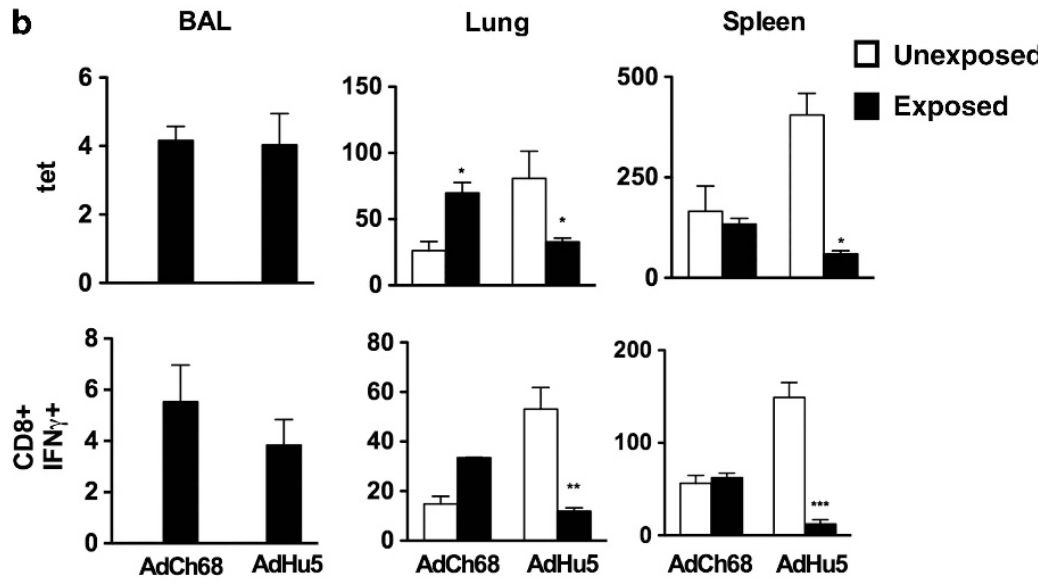

80
$60-1$
$40-1$
20

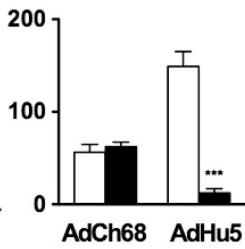

Absolute number per organ $\left(10^{3}\right)$

d

BAL
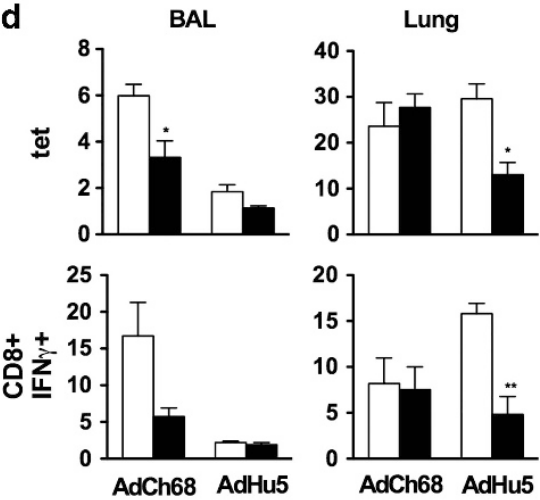

Absolute number per organ $\left(10^{3}\right)$

f
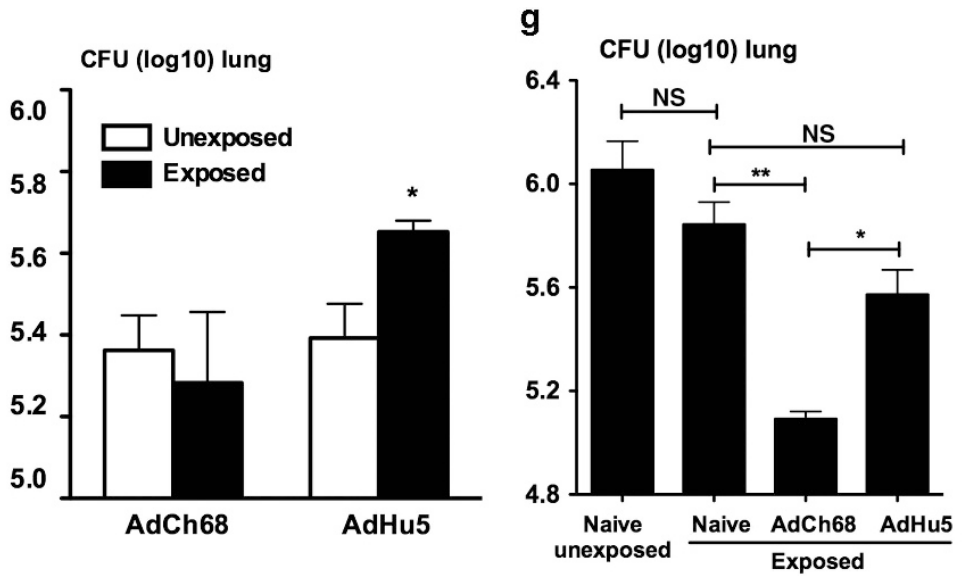

Figure 6 Potent protective immunity against pulmonary tuberculosis (TB) by respiratory mucosal AdCh68Ag85A immunization in hosts with preexisting anti-AdHu5 immunity in the lung. (a) Experimental schema for (b). Mice were exposed intranasally to wild-type AdHu5 virus (WtAd). The control mice were not exposed to WtAd (unexposed). At week 4 after WtAd exposure, mice were immunized intramuscularly with $1 \times 10^{7}$ plaque-forming units (PFUs) AdCh68Ag85A (AdCh68) or AdHu5Ag85A (AdHu5) vaccine. Bronchoalveolar lavage (BAL), lungs, and spleens were collected for analysis at week 2 after parenteral immunization. (b) Bar graphs show absolute numbers of Ag85A tetramer + and CD8 + IFN- $\gamma+\mathrm{T}$ cells in the airway lumen (BAL), lung interstitium (Lung), and spleen. IFN $\gamma$, interferon- $\gamma$. (c) Experimental schema for (d). Mice were exposed intranasally to WtAd. The control mice were not exposed to WtAd (unexposed). At week 4 after WtAd exposure, mice were immunized intranasally with AdCh68 or AdHu5 TB vaccine. BAL and lungs were collected for analysis at week 4 after respiratory mucosal immunization. (d) Bar graphs show absolute numbers of Ag85A tetramer + and CD8 + IFN- $\gamma+$ T cells in BAL and lung. (e) Experimental schema for $(\mathbf{f}, \mathbf{g})$. Mice were set up as described in $\mathbf{c}$ except that at week 4 after respiratory mucosal immunization, animals were challenged with Mycobacterium tuberculosis (M.tb) and the levels of infection in the lung were assessed 4 weeks after M.tb challenge. (f) Bar graph shows the colony-forming units (CFUs) of M.tb in the lung. (g) Mice were set up as described in e except that a group of mice exposed to WtAd but not immunized was included. Bar graph shows the CFUs of $M$.tb in the lung. Data in $\mathbf{b}, \mathbf{d}$ are expressed as the mean $\pm \mathrm{s}$.e.m. from three to four mice per group, representative of three independent experiments. Data in $f$ are the mean \pm s.e.m. from four to five mice per group. ${ }^{\star} P<0.05$; ${ }^{\star \star} P<0.01 ;{ }^{* \star} P<0.001$; NS, not significant $P>0.05$ compared with the unexposed control. 
a

4 Weeks

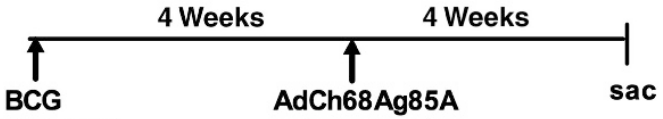

b

BAL

CD4+IF $\mathrm{N} \gamma+$ cells $\left(10^{3}\right)$

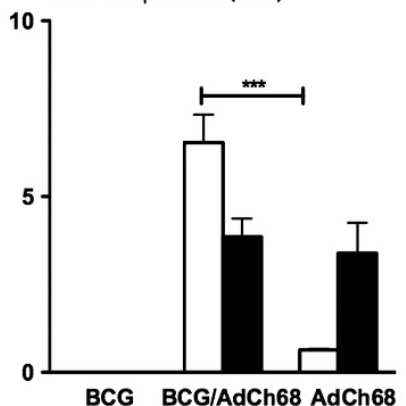

BCG BCG/AdCh68 AdCh68

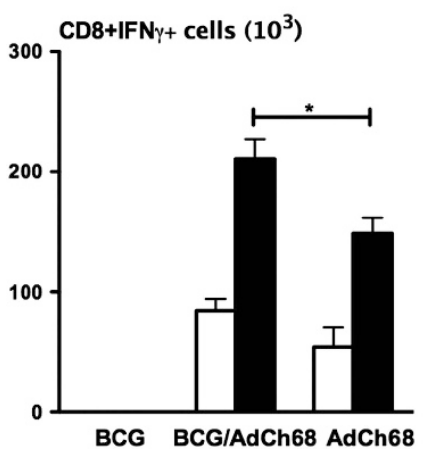

subcutaneous

C CD4+IFN $\gamma+$ cells $\left(10^{3}\right)$

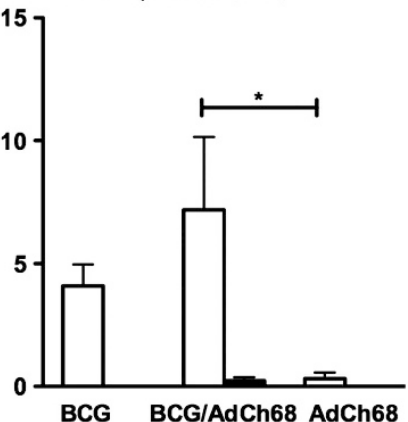

CD8+IFN $\gamma+$ cells $\left(10^{3}\right)$

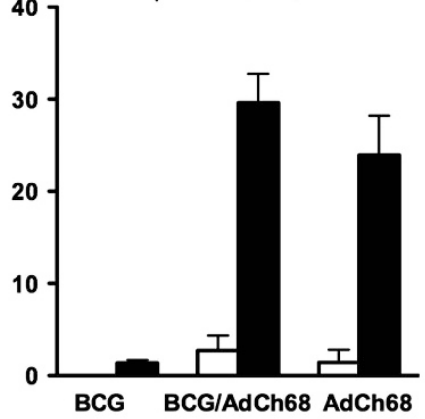

d

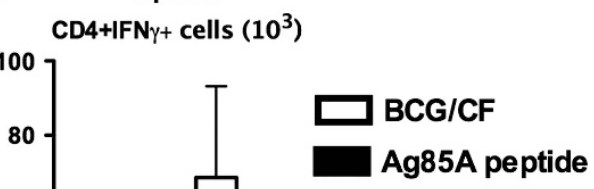

e

\section{Weeks}

4 Weeks
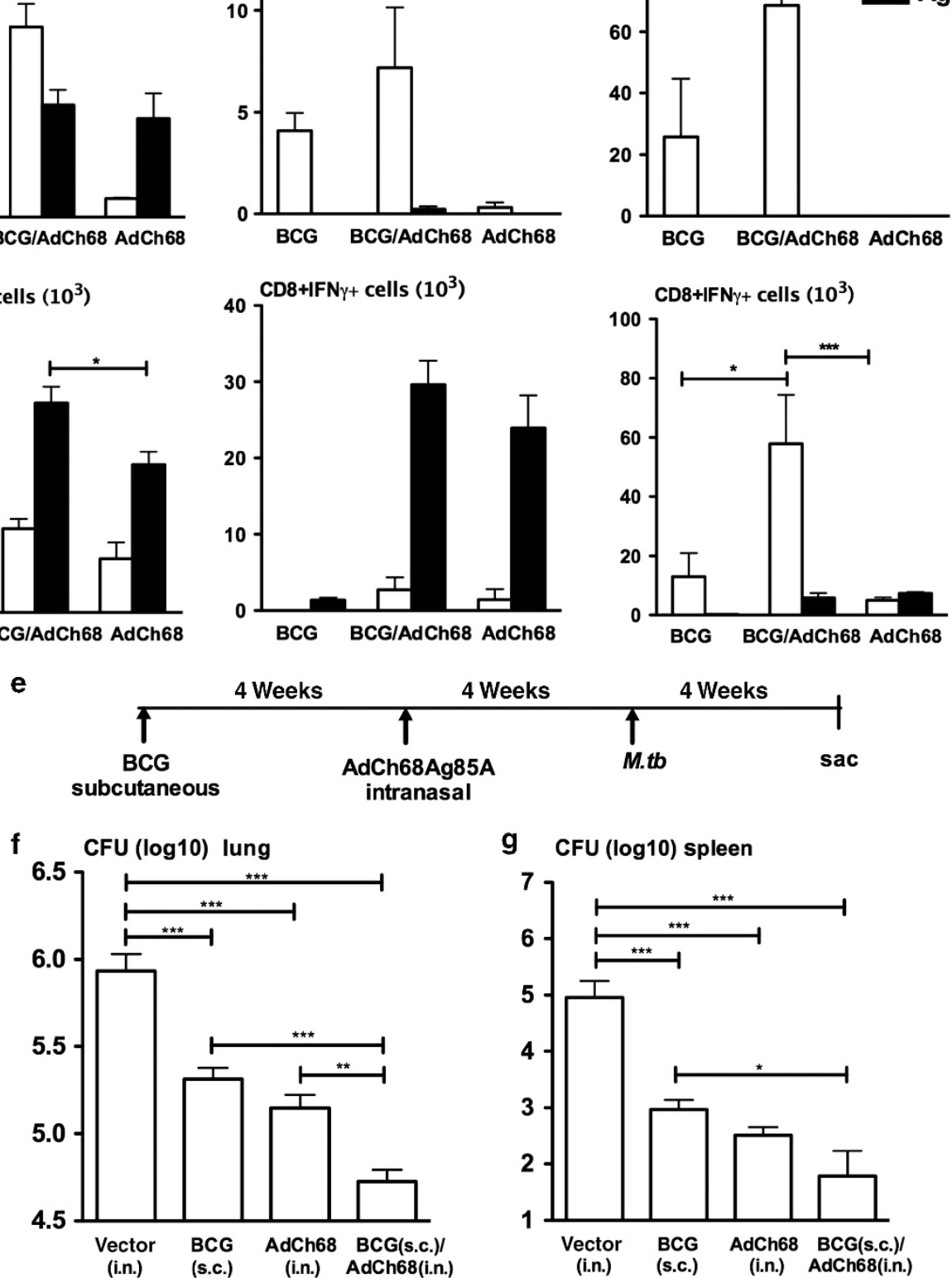

g CFU $(\log 10)$ spleen

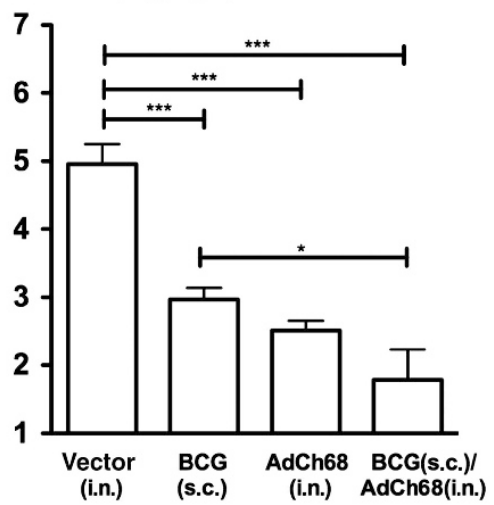

Figure 7 Further enhanced protective immunity against pulmonary tuberculosis (TB) by respiratory mucosal AdCh68Ag85A boost immunization in parenteral Bacillus Calmette-Guérin (BCG)-primed hosts. (a) Experimental schema for (b-d). Mice were prime-immunized subcutaneously (s.c.) with BCG. At week 4, animals were boost-immunized via intranasal (i.n.) route with $5 \times 10^{7}$ plaque-forming units (PFUs) AdCh68Ag85A (BCG/AdCh68). Control groups were immunized only with BCG (BCG) or AdCh68Ag85A (AdCh68). Bronchoalveolar lavage (BAL), lungs, and spleens were collected at week 4 after boost immunization. (b-d) Bar graphs show absolute numbers of CD4 + IFN- $\gamma+$ and CD8 + IFN- $\gamma+$ T cells in (b) BAL, (c) lung, and (d) spleen. The cells were stimulated either with crude mycobacterial antigens (BCG/culture filtrate (CF)) or an Ag85A CD4 or CD8 peptide (Ag85A peptide). (e) Experimental schema for (f, $\mathbf{g})$. Mice were set up as in a including BCG s.c., AdCh68 i.n., and BCG (s.c.)/AdCh68 (i.n.) groups except that at week 4 after boost immunization, mice were challenged with Mycobacterium tuberculosis (M.tb) and the lungs and spleens were collected for analysis. Furthermore, an additional control group was set up, receiving i.n. an empty control AdCh68 vector (vector). (f, $\mathbf{g}$ ) Bar graphs show the colony-forming units (CFUs) of $\mathbf{M}$.tb in the (f) lung and ( $\mathbf{g})$ spleen. Data in $\mathbf{b}, \mathbf{c}, \mathbf{d}$ are expressed as the mean \pm s.e.m. from three to four mice per group, representative of two independent experiments. Data in $\mathbf{f}, \mathbf{g}$ are the mean \pm s.e.m. from five mice per group, representative of two independent experiments. ${ }^{\star} P<0.05$; ${ }^{\star \star} P<0.01 ;{ }^{* \star \star} P<0.001$. 
cells isolated from the airway lumen (BAL), lung interstitium (lung), and spleen were stimulated with crude mycobacterial antigens (BCG/culture filtrate) or an Ag85A peptide and analyzed for antigen-specific, IFN $\gamma$-producing CD4 and CD8 T-cell responses. Consistent with our previous findings, ${ }^{8}$ parenteral BCG priming alone did not elicit airway luminal $\mathrm{CD} 4$ or CD8 $\mathrm{T}$-cell responses (Figure $7 \mathbf{b}$ ), whereas it induced a level of crude mycobacterial antigen (BCG/culture filtrate)reactive $\mathrm{CD} 4 \mathrm{~T}$-cell, but not $\mathrm{CD} 8 \mathrm{~T}$-cell, responses in the lung (Figure 7c) and spleen (Figure 7d). On the other hand, as expected, intranasal AdCh68 vaccination alone primarily activated Ag85A-specific CD8 $\mathrm{T}$ cells in the BAL and lung (Figure $7 \mathbf{b}, \mathbf{c}$ ). In comparison, intranasal AdCh68 boost vaccination of parenteral BCG-primed animals (BCG/ AdCh68) markedly increased multi-mycobacterial antigenspecific $\mathrm{CD} 4 \mathrm{~T}$ cells in the airway lumen (BAL), lung, and spleen (Figure $7 \mathbf{b}-\mathbf{d}$ ). It also increased both multimycobacterial antigen- and Ag85A-specific CD8 T cells in the BAL and spleen (Figure $\mathbf{7 b}-\mathbf{d}$ ). Together, these results indicate that respiratory mucosal AdCh68Ag85A boost immunization can potently enhance CD4 and CD8 T-cell responses in the lung in parenteral BCG-primed animals.

To investigate whether intranasal AdCh68Ag85A-boosted T cell immunity in the lung of BCG-primed hosts could lead to improved immune protection against pulmonary $\mathrm{TB}$, mice were subcutaneous BCG primed and received an intranasal boost with AdCh68 (BCG s.c./AdC68 i.n.) as above and subsequently challenged via the airway with virulent M.tbH37Rv (Figure 7e). Control groups were treated either with BCG alone (BCG s.c.) or AdCh68Ag85A alone (AdCh68 i.n.) or an empty AdCh68 virus alone (vector i.n.). Relative levels of protective efficacy in the lung and spleen were assessed at 4 weeks after $M . t b$ infection. As expected, the vector control animals that were inoculated with the empty AdCh68 virus were not protected, having high levels of M.tb infection in the lung (Figure 7f), similar to the levels seen in infected naive animals (Figure 5a). Again, as shown in Figure 5a, subcutaneous BCG priming alone or intranasal AdCh68 vaccination alone each significantly enhanced protection, causing $0.7-0.8 \log$ M.tb colony-forming unit (CFU) reduction (Figure 7f). However, correlating with its improved T-cell immunity (Figure 7b-d), AdCh68 boost vaccination of BCGprimed animals further improved protection, leading to an $\sim 1.4 \log$ M.tb CFU reduction in the lung (Figure 7f). Similarly, intranasal AdCh68 boosting also resulted in the best levels of improved protection in the spleen (3log M.tb CFU reduction) compared with vector, BCG, or AdCh68 alone (Figure 7g). The above data together establish that respiratory mucosal AdCh68Ag85A boost immunization markedly enhances antiTB T-cell immunity in parenteral BCG-primed hosts.

\section{DISCUSSION}

Mounting evidence suggests that any effective TB vaccination strategies ought to be able to close the "immunological gap" instigated by M.tb infection whereby the appearance of T-cell immunity is much delayed in the lung. ${ }^{6,7,11,12}$ Respiratory mucosal vaccination represents a powerful way to install the respiratory mucosa with T-cell immunity before $M$. $t b$ exposure for enhanced lung protection. ${ }^{6,11-14}$ The potency of respiratory mucosal vaccination is not only limited to intracellular infections as its advantage has also recently been recognized for immunity against lung cancer. ${ }^{49}$ Indeed, TB vaccine research community has recently identified the development of safe and effective respiratory mucosal TB vaccination strategies to be a new priority. ${ }^{20}$ Such renewed effort is timely as parenteral boosting with a vaccinia virus (MVA)-based TB vaccine was unable to improve lung protection in BCG vaccines as recently shown in a phase $2 \mathrm{~b}$ efficacy trial, the first of its kind to test a new TB vaccine in the world. ${ }^{19}$

Given its natural tropism to the respiratory mucosa and potency, recombinant AdHu5-based vaccine platforms have proven to be most effective and promising for respiratory mucosal vaccination against pulmonary TB based on abundant preclinical data. ${ }^{11,41,42,45,50-52}$ However, the high prevalence of preexisting anti-AdHu5 immunity in human populations has become an increasingly recognized limitation and challenge to application of AdHu5-based vaccines in humans. ${ }^{22,24,25,28-30,32}$ We have recently reported that preexisting circulating antiAdHu5 antibodies had no major effect on T-cell activation by intramuscularly administered AdHu5Ag85A vaccine in a human study. ${ }^{27}$ Nevertheless, as all trial volunteers had varying degrees of preexisting anti-AdHu5 antibodies, ${ }^{27}$ we cannot firmly conclude that responses to the vaccine were not dampened. Furthermore, as wild-type AdHu 5 virus is primarily a human respiratory pathogen, the anti-AdHu5 immunity concentrated in lung mucosa could have greater negative effects on respiratory-mucosally administered AdHu5-based vaccine. On the other hand, the results from Step HIV vaccine trials have implied a safety concern in using AdHu5-based vaccine in those HIV high-risk populations who have high levels of preexisting anti-AdHu5 immunity. ${ }^{32,53,54}$ Thus, despite the big strides made in basic and preclinical development of new TB vaccines, there exists an important gap in meeting with the conditions and effective applications in humans. These considerations have prompted recently accelerated pace in the development of heterologous chimpanzee adenovirus-based vaccine technology. $22,24,28,33,35$

In this study, by using a new technology ${ }^{40}$ we have developed the first chimpanzee adenovirus-vectored TB vaccine as a novel candidate for respiratory mucosal immunization in humans. We find that the ability of a single respiratory mucosal AdCh68Ag85A immunization to activate $\mathrm{T}$ cells, provide mucosal protection, and reduce M.tb-associated immunopathology in naive animals with or without parenteral BCG priming is comparable to or even better than AdHu5Ag85A counterpart. Of importance, we provide compelling evidence that contrary to AdHu5Ag85A, respiratory mucosal AdCh68Ag85A-induced protective T-cell immunity is minimally affected by anti-AdHu5 immunity preexisting locally in the lung. Given the scarcity of candidate TB vaccines currently amenable to safe and effective respiratory mucosal vaccination in humans, ${ }^{20}$ our study holds important implications. The 
findings from our study suggest that AdCh68-based TB vaccine is superior to the AdHu5-based counterpart and should be further developed clinically as an ideal TB vaccine candidate for respiratory mucosal immunization in humans. Given the ample experience and infrastructure well established by us and others, its expeditious translation to human studies and applications is expected.

Another noticeable advantage associated with AdCh68Ag85A vaccine is its broadened CD8 T-cell epitope specificity noted in our study. Thus, respiratory mucosal AdCh68Ag85A immunization results in the emergence of a dominant CD8 T-cell epitope corresponding to Ag85A amino acids 146-154 (Ep-2) in addition to its strong inducibility of CD8 T-cell responses to the epitope spanning amino acids 70-80 (Ep-1). In comparison, its AdHu5 counterpart induces a strong response only to Ep-1, with Ep-2 being a subdominant CD8 T-cell epitope. A broadened spectrum of dominant T-cell epitopes including Ep-2 by AdCh68-based immunization is considered an additional benefit to human applications as the epitopes within Ag85A amino acids 141-160 were previously found to be the most immunodominant and MHC promiscuous in M.tb-infected humans. ${ }^{55}$ We believe that such broadened T-cell epitope specificity by AdCh68-based immunization may have contributed to improved protection over that by AdHu5Ag85A immunization, particularly with regard to reduced lung immunopathology. At the present time, the mechanisms for such difference between AdCh and AdHu5 vectors still remain to be fully understood. Although both AdCh and AdHu5 utilize the same coxsackievirus and adenovirus receptor for infection, ${ }^{24,56}$ and we have observed their proinflammatory properties to be comparable, it is possible that other differentially regulated intracellular signaling pathways may play a role. Indeed, previous studies suggest that the nature of formulation or vaccine vector has a profound effect on the immunodominance of T-cell epitopes and the immune protective potential of activated $\mathrm{T}$ cells. ${ }^{57,58}$ This could be attributed to antigen processing and presentation by antigenpresenting cells to $\mathrm{CD} 8 \mathrm{~T}$ cells regulated by genes expressed by the vector. ${ }^{59,60}$ Thus, the broaden epitope recognition following AdCh vaccination could be explained in part by the differential inherent prosperities of the viral vectors having a role in antigen processing and canonical epitope presentation. However, whether the broadened epitope specificity of AdCh68Ag85A vaccine may also hold true in humans requires further investigation. Of note, despite enhanced T-cell responses by AdCh immunization relative to AdHu5 immunization, the enhanced bacterial control by the two is comparable in WtAdunexposed hosts. This suggests that further improved T-cell responses may not always translate into further improved mycobacterial control. Nonetheless, such further improved T-cell responses by AdCh immunization did lead to improved protection with respect to M.tb-associated histopathology.

In keeping with previously published studies, ${ }^{24,38}$ we find that in contrast to AdHu5 vaccine the potency of parenterally (intramuscularly) administered AdCh vaccine is not negatively affected at all by preexisting systemic anti-AdHu5 immunity. This finding supports that anti-AdHu5 antibodies do not crossreact with AdCh viral vectors. However, it is noteworthy that in the majority of previous studies, preexisting anti-AdHu5 immunity was generated by systemic AdHu5 infection. As AdHu5 virus is a respiratory pathogen in humans and recent evidence suggests a differential immune-regulatory effect by the mucosal route of induction of anti-AdHu5 immunity, ${ }^{61,62}$ in this study we generated preexisting anti-AdHu5 immunity by respiratory route of wild-type AdHu 5 infection that generated anti-Ad5-neutralizing antibody titers in both the lung and circulation, similar to the levels seen in humans. ${ }^{62}$ Thus, this method is not only clinically relevant but is also relevant to the investigation of AdCh-based respiratory mucosal $\mathrm{TB}$ vaccination strategies. Recently emerging clinical evidence has also raised the question of whether anti-AdHu5 $\mathrm{T}$ cells may crossreact with the antigens from AdCh infection, ${ }^{30,32,35,63}$ thus potentially negatively affecting the protective immunity by AdCh-based immunization. Indeed, we find that preexisting anti-AdHu5 immunity present in the lung does have a dampening effect on AdCh68-induced T-cell responses within the airway lumen. However, this dampening effect was found to be limited only to the airway luminal space as the antigenspecific $\mathrm{T}$-cell responses within the lung interstitium were not affected. In contrast, preexisting local anti-AdHu5 immunity significantly reduced the $T$ cells in the lung interstitium of AdHu5-vaccinated hosts. Thus, despite somewhat dampened $\mathrm{T}$-cell responses in the airway lumen by local anti-AdHu5 immunity, the lungs of AdCh68Ag85A-vaccinated hosts retained much greater numbers of $M . t b$ antigen-specific $\mathrm{T}$ cells than AdHu5Ag85A-vaccinated counterparts. This underpins one of the most important findings in our study that preexisting anti-AdHu5 immunity in the lung does not negatively affect AdCh68Ag85A-induced immune protection against pulmonary $\mathrm{TB}$, whereas it significantly reduces AdHu5Ag85A-mediated protection. In this study we have also demonstrated a dramatic boosting effect by AdCh68Ag85A immunization in BCG-primed animals. Respiratory mucosal AdCh68 immunization boosted both multi-mycobacterial antigen-specific CD4 T cells and Ag85A-specific CD8 T cells that led to markedly further enhanced protection.

In summary, we have developed a novel chimpanzee adenovirus-based TB vaccine for respiratory mucosal immunization and evaluated its potency in animal models of human pulmonary $\mathrm{TB}$ with or without preexisting anti-AdHu5 immunity in the lung. We provide compelling evidence that chimpanzee adenovirus-based $\mathrm{TB}$ vaccine is superior to AdHu5-based counterpart and thus may represent an ideal candidate for respiratory mucosal immunization in humans. Our findings also hold important implications in developing effective virus-based vaccines against other respiratory infection diseases and cancers for human applications.

\section{METHODS}

Animals for in vivo models of immunization and pulmonary tuberculosis. Female BALB/c mice, 6 to 8 weeks old, were purchased from Charles River Laboratories (Charles River, St Constant, Quebec, Canada) and housed in a specific pathogen-free level B facility at 
McMaster University. All experiments were carried out in accordance with the guidelines from the Animal Research and Ethics Board at McMaster University.

Bioengineering of human and chimpanzee adenovirus-based tuberculosis vaccines. The construction of a recombinant replication-deficient human type 5 adenovirus expressing an immunodominant $M . t b$ antigen 85A (AdHu5Ag85A) has been previously described. ${ }^{41,64}$ This vaccine has been extensively evaluated as a parenteral or respiratory mucosal $\mathrm{TB}$ vaccine in a wide range of preclinical models. ${ }^{41,42,45,50-52}$ It has also recently been evaluated in a phase 1 human study. ${ }^{27}$

A replication-deficient chimpanzee type 68 adenovirus was constructed to express an immunodominant M.tb antigen $85 \mathrm{~A}$ (AdCh68Ag85A) by using a direct cloning technology. ${ }^{40}$ The genetic Ag85A cassette containing the murine cytomegalovirus promoter, tissue plasminogen activator peptide signal, and polyA sequences used for constructing AdCh68Ag85A (Figure 1a) is identical to the one for AdHu5Ag85A. Briefly, a pShuttle plasmid DNA containing the Ag85A cassette was digested with I-Ceul and PI-Sce1. The released insert was subcloned by an in-gel ligation technique into the I-Ceu1 and PI-Sce1 site of the genomic DNA clone pAdC68 containing the entire AdCh68 genomic sequences except the E1 and E3 genes. AdCh68Ag85A was packaged and propagated in 293 cells and purified by cesium chloride gradient centrifugation in the same way as for AdHu5Ag85A. The Ag85A protein production and secretion by AdCh68Ag85A-infected mammalian cells was verified and compared with that in AdHu5Ag85A-infected cells by western immunoblotting using a monoclonal antibody specific for Ag85A (Figure 1b). ${ }^{41}$

Generation of preexisting anti-AdHu5 immunity in animals by respiratory infection with wild-type human serotype 5 adenovirus. In some experiments, naive mice were infected i.n. with WtAd $\left(1 \times 10^{8}\right.$ plaque-forming units (PFUs)) to generate preexisting anti-AdHu5 immunity before TB immunization.

Respiratory mucosal or parenteral immunization in animals with AdCh68Ag85A, AdHu5Ag85A, or BCG vaccine. Respiratory mucosal route of immunization was carried out by i.n. delivery of AdHu5Ag85A or AdCh68Ag85A $\left(1 \times 10^{7}\right.$ PFUs per mouse). ${ }^{42,45,50}$ In selected experiments, mice were immunized parenterally (intramuscular) with the same dose of vaccine. In some experiments a larger dose $\left(5 \times 10^{7} \mathrm{PFUs}\right)$ of AdCh68Ag85A was used. For prime-boost immunization regimen, BCG (Pasteur) $\left(1 \times 10^{5}\right.$ CFUs per mouse) was inoculated s.c. ${ }^{50}$ for priming and the animals were then boosted i.n. with Ad-based vaccine.

M. tuberculosis preparation and animal models of pulmonary tuberculosis. M.tb bacilli were grown in Middlebrook 7H9 broth supplemented with Middlebrook oleic acid-albumin-dextrose-catalase enrichment, $0.002 \%$ glycerol, and $0.05 \%$ Tween- 80 for $10-15$ days, aliquoted, and stored in $-70^{\circ} \mathrm{C}$ until use. ${ }^{41,42,45,50}$ Before each in vivo use, $M . t b$ bacilli were washed with phosphate-buffered saline containing $0.05 \%$ Tween- 80 twice and passed through a 27 -gauge needle 10 times to disperse clumps. Pulmonary infection with M.tbH37Rv strain was carried out as previously described. ${ }^{41,42,45,50}$ The levels of mycobacterial infection in the lung and spleen were determined by plating serial dilutions of tissue homogenates in triplicates onto Middlebrook 7H10 agar plates. Plates were incubated at $37^{\circ} \mathrm{C}$ for $15-17$ days before colony enumeration.

Bronchoalveolar lavage and lung and spleen mononuclear cell isolation. Mice were killed by exsanguination. Airway luminal cells were collected through exhaustive BAL as previously described. ${ }^{42,45}$ Subsequently, lungs were perfused by injecting Hank's buffer through the right ventricle in order to remove intravascular mononuclear cells. Lungs were digested with collagenase type 1 (Sigma-Aldrich, St Louis, $\mathrm{MO}$ ) at $37^{\circ} \mathrm{C}$ in an agitating incubator. A single-cell suspension was obtained by crushing the digested tissue through $40 \mu \mathrm{m}$ basket filter.
Splenocytes were isolated as described previously. ${ }^{41,50}$ All isolated cells were resuspended in complete RPMI-1640 medium (RPMI 1640 supplemented with $10 \%$ fetal bovine serum, $1 \%$ penicillinstreptomycin, and 1\% L-glutamine).

Cell stimulation, tetramer staining, intracellular cytokine staining, and flow cytometry. Mononuclear cells from BAL, lungs, or spleens were cultured in U-bottom 96-well plates at a concentration of 20 million cells per $\mathrm{ml}$ for lungs and spleens and 0.5 million cells per $\mathrm{ml}$ for BAL. For intracellular cytokine staining, ${ }^{42,45,50}$ cells were cultured in the presence of Golgi plug ( $5 \mathrm{mg} \mathrm{ml}^{-1}$ brefeldin A; BD Pharmingen, San Jose, CA) with or without stimulation for 5-6 h with an Ag85Aspecific CD8 T cell-specific peptide (MPVGGQSSF)-Ep-1, a newly identified Ag85A-specific CD8 T-cell peptide (VYAGAMSGL)-Ep-2, or Ag85A-specific CD4 peptide (LTSELPGWLQANRHVKPTGS) at a concentration of $1 \mu \mathrm{g}$ per well. In selected experiments, cells were stimulated with crude BCG and M.tb CF at a concentration of $1 \mu \mathrm{g}$ per well for $24 \mathrm{~h}$ with last $6 \mathrm{~h}$ in the presence of Golgi plug. After incubation, cells were washed and blocked with CD16/CD32 in $0.5 \%$ bovine serum albumin/phosphate-buffered saline for $15 \mathrm{~min}$ on ice and then stained with the appropriate fluorochrome-labeled monoclonal antibodies. Cells were then processed according to the manufacturer's instructions (BD Pharmingen). The monoclonal antibodies used included CD8a-phycoerythrin-Cy7, CD4-allophycocyanin-Cy7, IFN- $\gamma$-allophycocyanin, and CD3-CyChrome. For tetramer immunostaining, a tetramer for the immunodominant CD8 T-cell peptide (MPVGGQSSF) of Ag85A bound to the BALB/c major histocompatibility complex class I allele H-2L ${ }^{\mathrm{d}}$ (NIH Tetramer Core, Atlanta, GA) was used. Cells were washed and blocked with CD16/ CD32 in $0.5 \%$ bovine serum albumin/phosphate-buffered saline for $15 \mathrm{~min}$ on ice and stained with the tetramer for $1 \mathrm{~h}$ in the dark at room temperature. Cells were then washed and stained with surface antibodies. Immunostained cells were run on an LSR II flow cytometer (BD Biosciences, San Jose, CA) and 250,000 events per sample were collected and analyzed on FlowJo software (version 9; Tree Star, Ashland, OR).

T-cell epitope mapping for identification of a newly emerged T-cell epitope following AdCh68Ag85A immunization. To investigate the repertoire of antigen-specific CD8 T cells generated by AdC68Ag85A and AdHu5Ag85A immunization, lung mononuclear cells from immunized mice were ex vivo stimulated with pools of 57 peptides of Ag85 $\mathrm{A}^{27}$ by using a previously described method. ${ }^{47}$ In the initial screening, lung cells were stimulated separately with six peptide pools (p1-p10, p11-p20, p21-p30, p31-p40, p41-p50, and p51-p57; each peptide is 15 amino acids in length with 10 amino acids overlapping) or single peptide pool containing 1-57 peptides. Each peptide in every pool was kept at a concentration of $1 \mu \mathrm{g}$ per well. Based on initial screening, two peptide pools (p21-p30 and p41-p50) containing potential CD8 T-cell epitopes were selected to design 9 sets of new peptide pools containing $2-9$ peptides/pool in such a way that each peptide is present only in 3 unique pools. These peptide pools were then used for ex vivo stimulation of lung mononuclear cells from immunized mice. Based on the magnitude of T-cell reactivity, a 15amino-acid peptide potentially containing an immunodominant CD8 T-cell epitope was identified and the corresponding amino acid sequence was analyzed using the Immune Epitope Database (http:// tools.immuneepitope.org/) for determining the highest probable CD8 T-cell epitope sequence. A novel Ag85A-specific CD8 T cell-specific epitope sequence (VYAGAMSGL) was identified and the corresponding peptide synthesized and used for ex vivo antigen stimulation as described above.

In vivo intratracheal cytotoxic T-cell assay. The intratracheal in vivo CD8 CTL assay was conducted as previously described. ${ }^{45}$ Briefly, $2.5 \times 10^{6}$ antigen-pulsed and $2.5 \times 10^{6}$ unpulsed, CFSE (carboxyfluorescein succinimidyl ester)-labeled splenic CTL target cells were combined to a final $40 \mu \mathrm{l}$ of phosphate-buffered saline and transferred 
intratracheally to the airway of mice vaccinated with Ad-based vaccine for 2 or 4 weeks. The animals were killed at $5 \mathrm{~h}$ after target cell transfer. Total cells isolated by bronchoalveolar lavage were run on the FACScan (BD Pharmingen) for assessment of the percentage of CFSElabeled target cells. The percent in vivo killing of CFSE-labeled target cells was defined as the relative loss of such cells after in vivo incubation and thus was taken as the measure of CTL.

Assessment of lung inflammation by histology, BAL cytology, and innate cytokine responses. Lung inflammation following respiratory mucosal immunization with AdCh68Ag85A or AdHu5Ag85A was assessed by blinded lung histopathologic examination, BAL differential inflammatory cell counting, and BAL cytokine measurement as previously described. ${ }^{41,42,45}$ The levels of BAL cytokine proteins including tumor necrosis factor- $\alpha$ and IFN $\beta$ were measured by enzyme linked immunosorbent assay (ELISA; R\&D Systems, Burlington, ON, Canada).

ELISA for measuring anti-AdHu5 IgG1, IgG2A, and IgA antibodies. Levels of anti-AdHu5-specific antibodies in BAL fluids and sera were determined by ELISA as previously described. ${ }^{65}$ Briefly, Nunc immunoplates were coated with $50 \mu \mathrm{l}$ per well of wild-type AdHu5 virus-infected HeLa cell lysate $\left(100 \mu \mathrm{g} \mathrm{ml}^{-1}\right)$ overnight at $4{ }^{\circ} \mathrm{C}$. Serial dilutions of sera or BAL fluids were set up in duplicates and incubated at $37^{\circ} \mathrm{C}$ for $1 \mathrm{~h}$. Goat anti-mouse IgG1-biotin or IgG2a-biotin (Southern Biotechnology Associates, Birmingham, AL) or IgA-biotin conjugates (Sigma-Aldrich) were added and incubated for $1 \mathrm{~h}$ at $37^{\circ} \mathrm{C}$, followed by streptavidin-conjugated alkaline phosphatase (R\&D Systems, Minneapolis, MN) for $1 \mathrm{~h}$ at $37^{\circ} \mathrm{C}$. The substrate was added to each well and incubated at room temperature for 15 to $30 \mathrm{~min}$ in the dark. After stoppage of reaction, the colorimetric change was evaluated with a microplate reader at $405 \mathrm{~nm}$.

Anti-WtAdHu5-neutralizing antibody measurement. The amount of neutralizing antibody titers present in serum or BAL was determined according to established methods. ${ }^{27}$ Samples were serially diluted in culture media starting from neat. Appropriate controls were set up with serum or BAL samples from naive mice (negative) or with just media alone (positive). Each dilution was mixed with AdHu5expressing Escherichia coli $\beta$-galactosidase (multiplicity of infection of 5), incubated for $1 \mathrm{~h}$ at $37^{\circ} \mathrm{C}$, and applied to confluent A549 epithelial cells in 96-well plates overnight at $37^{\circ} \mathrm{C}$. Neutralizing antibody titers were enumerated with AxioVision4 software (Carl Zeiss, Berlin, Germany). Wt-AdHu5-neutralizing antibody titers were calculated as $1 /(($ number of stained cells $\times$ total number of quadrants $) /$ $\mathrm{PFU}) \times$ sera/BAL dilution.

Data analysis. The differences deemed statistically significant were indicated as ${ }^{*} P<0.05,{ }^{*} P<<0.01$, and ${ }^{*}{ }^{*} P<0.001$. A two-tailed Student's $t$-test was performed for pair-wise comparisons. One-way analysis of variance and subsequent Tukey's post hoc test were carried out for the comparison of multiple groups. All analyses were performed by using GraphPad Prism software (GraphPad Software, San Diego, CA).

SUPPLEMENTARY MATERIAL is linked to the online version of the paper at http://www.nature.com/mi

\section{ACKNOWLEDGMENTS}

This work was supported by funds from the Canadian Institutes of Health Research, the Natural Sciences and Engineering Research Council of Canada, the Canadian Foundation for Innovation, and Ontario Government. We are grateful to the technical advice provided by Dr Xiangyang Zhou. We also acknowledge the kind provision of purified M.tb antigens, anti-Ag85A monoclonal antibodies, and Ag85A MHC class I tetramers by the BEI Resources, Kris Huygen, and the NIH Tetramer Core, respectively.

\section{DISCLOSURE}

The authors declared no conflict of interest. c 2015 Society for Mucosal Immunology

\section{REFERENCES}

1. Cooper, A.M. Cell-mediated immune responses in tuberculosis. Annu. Rev. Immunol. 27, 393-422 (2009).

2. Kaufmann, S.H. Tuberculosis vaccine development: strength lies in tenacity. Trends Immunol. 33, 373-379 (2012).

3. McShane, H. et al. BCG: myths, realities, and the need for alternative vaccine strategies. Tuberculosis (Edinb) 92, 283-288 (2012).

4. Brennan, M.J., Stone, M.R. \& Evans, T. A rational vaccine pipeline for tuberculosis. Int. J. Tuberc. Lung Dis. 16, 1566-1573 (2012).

5. Behr, M.A. BCG-different strains, different vaccines? Lancet Infect. Dis. 2, 86-92 (2002).

6. Xing, Z., Jeyanathan, M. \& Smaill, F. New approaches to TB vaccination. Chest 146, 804-812 (2014).

7. Urdahl, K.B., Shafiani, S. \& Ernst, J.D. Initiation and regulation of T-cell responses in tuberculosis. Mucosal Immunol. 4, 288-293 (2011).

8. Horvath, C.N., Shaler, C.R., Jeyanathan, M., Zganiacz, A. \& Xing, Z. Mechanisms of delayed anti-tuberculosis protection in the lung of parenteral BCG-vaccinated hosts: a critical role of airway luminal T cells. Mucosal. Immunol. 5, 420-431 (2012).

9. Jeyanathan, M. et al. Pulmonary M. tuberculosis infection delays Th1 immunity via immunoadaptor DAP12-regulated IRAK-M and IL-10 expression in antigen-presenting cells. Mucosal Immunol. 7, 670-683 (2014).

10. Chen, K. \& Kolls, J.K. T cell-mediated host immune defenses in the lung. Annu. Rev. Immunol. 31, 605-633 (2013).

11. Beverley, P.C., Sridhar, S., Lalvani, A. \& Tchilian, E.Z. Harnessing local and systemic immunity for vaccines against tuberculosis. Mucosal Immunol. 7, 20-26 (2014).

12. Jeyanathan, M., Heriazon, A. \& Xing, Z. Airway luminal Tcells: a newcomer on the stage of TB vaccination strategies. Trends Immunol. 31, 247-252 (2010).

13. Belyakov, I.M. \& Ahlers, J.D. What role does the route of immunization play in the generation of protective immunity against mucosal pathogens? J. Immunol. 183, 6883-6892 (2009).

14. Griffiths, K.L. \& Khader, S.A. Novel vaccine approaches for protection against intracellular pathogens. Curr. Opin. Immunol. 28C, 58-63 (2014).

15. Lycke, N. Recent progress in mucosal vaccine development: potential and limitations. Nat. Rev. Immunol. 12, 592-605 (2012).

16. Baaten, B.J., Cooper, A.M., Swain, S.L. \& Bradley, L.M. Location, location, location: the impact of migratory heterogeneity on $\mathrm{T}$ cell function. Front Immunol 4, 311 (2013).

17. Andersen, P. \& Kaufmann, S.H. Novel vaccination strategies against tuberculosis. Cold Spring Harb. Perspect. Med. 4, 210-216 (2014).

18. Lewis, D.J. et al. Transient facial nerve paralysis (Bell's palsy) following intranasal delivery of a genetically detoxified mutant of Escherichia coli heat labile toxin. PLoS One 4, e6999 (2009).

19. Tameris, M.D. et al. Safety and efficacy of MVA85A, a new tuberculosis vaccine, in infants previously vaccinated with BCG: a randomised, placebo-controlled phase 2b trial. Lancet 381, 1021-1028 (2013).

20. Aerosol Vaccines For Tuberculosis Workshop Summary Group: Developing Aerosol Vaccines for Mycobacterium tuberculosis. Vaccine, 2015 (in press).

21. Xing, Z. \& Lichty, B.D. Use of recombinant virus-vectored tuberculosis vaccines for respiratory mucosal immunization. Tuberculosis (Edinb) $\mathbf{8 6}$, 211-217 (2006).

22. Rollier, C.S., Reyes-Sandoval, A., Cottingham, M.G., Ewer, K. \& Hill, A.V. Viral vectors as vaccine platforms: deployment in sight. Curr. Opin. Immunol. 23, 377-382 (2011).

23. Song, K. et al. Genetic immunization in the lung induces potent local and systemic immune responses. Proc. Natl. Acad. Sci. USA 107, 2221322218 (2010).

24. Lasaro, M.O. \& Ertl, H.C. New insights on adenovirus as vaccine vectors. Mol. Ther. 17, 1333-1339 (2009).

25. Majhen, D. et al. Adenovirus-based vaccines for fighting infectious diseases and cancer: progress in the field. Hum. Gene Ther. 25, 301-317 (2014).

26. Sullivan, N.J. et al. CD8 + cellular immunity mediates rAd5 vaccine protection against Ebola virus infection of nonhuman primates. Nat. Med. 17, 1128-1131 (2011). 
27. Smaill, F. et al. A human type 5 adenovirus-based tuberculosis vaccine induces robust $T$ cell responses in humans despite preexisting antiadenovirus immunity. Sci. Transl. Med. 5, 205ra134 (2013).

28. Kaufmann, S.H., Juliana McElrath, M., Lewis, D.J. \& Del Giudice, G. Challenges and responses in human vaccine development. Curr. Opin. Immunol. 28C, 18-26 (2014).

29. Xiang, Z. et al. Chimpanzee adenovirus antibodies in humans, subSaharan Africa. Emerg. Infect. Dis. 12, 1596-1599 (2006).

30. Frahm, N. et al. Human adenovirus-specific T cells modulate HIV-specific T cell responses to an Ad5-vectored HIV-1 vaccine. J. Clin. Invest. 122 359-367 (2012).

31. Zak, D.E. et al. Merck Ad5/HIV induces broad innate immune activation that predicts $\mathrm{CD} 8(+)$ T-cell responses but is attenuated by preexisting Ad5 immunity. Proc. Natl. Acad. Sci. USA 109, E3503-E3512 (2012).

32. Fauci, A.S., Marovich, M.A., Dieffenbach, C.W., Hunter, E. \& Buchbinder, S.P. Immunology. Immune activation with HIV vaccines. Science 344, 49-51 (2014).

33. Colloca, S. et al. Vaccine vectors derived from a large collection of simian adenoviruses induce potent cellular immunity across multiple species. Sci. Transl. Med. 4, 115ra112 (2012).

34. Quinn, K.M. et al. Comparative analysis of the magnitude, quality, phenotype, and protective capacity of simian immunodeficiency virus gag-specific CD8 + T cells following human-, simian-, and chimpanzeederived recombinant adenoviral vector immunization. J. Immunol. 190, 2720-2735 (2013)

35. Capone, S. et al. Development of chimpanzee adenoviruses as vaccine vectors: challenges and successes emerging from clinical trials. Expert Rev. Vaccines 12, 379-393 (2013).

36. Barnes, E. et al. Novel adenovirus-based vaccines induce broad and sustained T cell responses to HCV in man. Sci. Transl. Med. 4, 115ra111 (2012).

37. Ewer, K.J. et al. Protective CD8 + T-cell immunity to human malaria induced by chimpanzee adenovirus-MVA immunisation. Nat. Commun. 4, 2836 (2013).

38. Fitzgerald, J.C. et al. A simian replication-defective adenoviral recombinant vaccine to HIV-1 gag. J. Immunol. 170, 1416-1422 (2003).

39. Kobinger, G.P. et al. Chimpanzee adenovirus vaccine protects against Zaire Ebola virus. Virology 346, 394-401 (2006)

40. Zhou, D. et al. An efficient method of directly cloning chimpanzee adenovirus as a vaccine vector. Nat. Protoc. 5, 1775-1785 (2010).

41. Wang, J. et al. Single mucosal, but not parenteral, immunization with recombinant adenoviral-based vaccine provides potent protection from pulmonary tuberculosis. J. Immunol. 173, 6357-6365 (2004).

42. Jeyanathan, M. et al. Differentially imprinted innate immunity by mucosal boost vaccination determines antituberculosis immune protective outcomes, independent of T-cell immunity. Mucosal Immunol. 6, 612-625 (2013).

43. Teles, R.M. et al. Type I interferon suppresses type II interferon-triggered human anti-mycobacterial responses. Science 339, 1448-1453 (2013).

44. Antonelli, L.R. et al. Intranasal Poly-IC treatment exacerbates tuberculosis in mice through the pulmonary recruitment of a pathogen-permissive monocyte/macrophage population. J. Clin. Invest. 120, 1674-1682 (2010).

45. Santosuosso, M., Zhang, X., McCormick, S., Wang, J., Hitt, M. \& Xing, Z. Mechanisms of mucosal and parenteral tuberculosis vaccinations: adenoviral-based mucosal immunization preferentially elicits sustained accumulation of immune protective CD4 and CD8 T cells within the airway lumen. J. Immunol. 174, 7986-7994 (2005).

46. D'Souza, S., Rosseels, V., Romano, M., Tanghe, A., Denis, O. \& Jurion, F. et al. Mapping of murine Th1 helper T-cell epitopes of mycolyl transferases Ag85A, Ag85B, and Ag85C from Mycobacterium tuberculosis. Infect. Immun. 71, 483-493 (2003).

47. Hoffmeister, B., Kiecker, F., Tesfa, L., Volk, H.D., Picker, L.J. \& Kern, F. Mapping T cell epitopes by flow cytometry. Methods 29, 270-281 (2003).
48. McCoy, K. et al. Effect of preexisting immunity to adenovirus human serotype 5 antigens on the immune responses of nonhuman primates to vaccine regimens based on human- or chimpanzee-derived adenovirus vectors. J. Virol. 81, 6594-6604 (2007).

49. Sandoval, F. et al. Mucosal imprinting of vaccine-induced CD8(+) Tcells is crucial to inhibit the growth of mucosal tumors. Sci. Transl. Med. 5 , 172ra120 (2013).

50. Santosuosso, M., McCormick, S., Zhang, X., Zganiacz, A. \& Xing, Z. Intranasal boosting with an adenovirus-vectored vaccine markedly enhances protection by parenteral Mycobacterium bovis BCG immunization against pulmonary tuberculosis. Infect. Immun. 74, 4634-4643 (2006).

51. Dean, G. et al. Comparison of the immunogenicity and protection against bovine tuberculosis following immunization by BCG-priming and boosting with adenovirus or protein based vaccines. Vaccine 32 , 1304-1310 (2014).

52. Xing, Z., McFarland, C.T., Sallenave, J.M., Izzo, A., Wang, J. \& McMurray, D.N. Intranasal mucosal boosting with an adenovirus-vectored vaccine markedly enhances the protection of BCG-primed guinea pigs against pulmonary tuberculosis. PLoS One 4, e5856 (2009).

53. Duerr, A. et al. Extended follow-up confirms early vaccine-enhanced risk of HIV acquisition and demonstrates waning effect over time among participants in a randomized trial of recombinant adenovirus HIV vaccine (Step Study). J. Infect. Dis. 206, 258-266 (2012).

54. Perreau, M., Pantaleo, G. \& Kremer, E.J. Activation of a dendritic cell-T cell axis by Ad5 immune complexes creates an improved environment for replication of HIV in T cells. J. Exp. Med. 205, 2717-2725 (2008).

55. Launois, P. et al. T-cell-epitope mapping of the major secreted mycobacterial antigen Ag85A in tuberculosis and leprosy. Infect. Immun. 62, 3679-3687 (1994).

56. Cohen, C.J., Xiang, Z.Q., Gao, G.P., Ertl, H.C., Wilson, J.M. \& Bergelson, J.M. Chimpanzee adenovirus CV-68 adapted as a gene delivery vector interacts with the coxsackievirus and adenovirus receptor. J. Gen. Virol. 83, 151-155 (2002).

57. Bennekov, T., Dietrich, J., Rosenkrands, I., Stryhn, A., Doherty, T.M. \& Andersen, P. Alteration of epitope recognition pattern in Ag85B and ESAT-6 has a profound influence on vaccine-induced protection against Mycobacterium tuberculosis. Eur. J. Immunol. 36, 3346-3355 (2006).

58. Tchilian, E., Ahuja, D., Hey, A., Jiang, S. \& Beverley, P. Immunization with different formulations of Mycobacterium tuberculosis antigen 85A induces immune responses with different specificity and protective efficacy. Vaccine 31, 4624-4631 (2013).

59. Goonetilleke, N. \& McMichael, A.J. Immunology. Antigen processing takes a new direction. Science 340, 937-938 (2013).

60. Hansen, S.G. et al. Cytomegalovirus vectors violate CD8 + T cell epitope recognition paradigms. Science 340, 1237874 (2013).

61. Choi, J.H., Schafer, S.C., Zhang, L., Juelich, T., Freiberg, A.N. \& Croyle, M.A. Modeling pre-existing immunity to adenovirus in rodents: immunological requirements for successful development of a recombinant adenovirus serotype 5-based ebola vaccine. Mol. Pharm. 10, 3342-3355 (2013).

62. Richardson, J.S., Abou, M.C., Tran, K.N., Kumar, A., Sahai, B.M. \& Kobinger, G.P. Impact of systemic or mucosal immunity to adenovirus on Ad-based Ebola virus vaccine efficacy in guinea pigs. J. Infect. Dis. 204, S1032-S1042 (2011).

63. Hutnick, N.A., Carnathan, D., Demers, K., Makedonas, G., Ertl, H.C. \& Betts, M.R. Adenovirus-specific human T cells are pervasive, polyfunctional, and cross-reactive. Vaccine 28, 1932-1941 (2010).

64. Xing, Z. et al. Recent advances in the development of adenovirus- and poxvirus-vectored tuberculosis vaccines. Curr. Gene Ther. 5, 485-492 (2005).

65. Xing, Z., Zganiacz, A., Wang, J., Divangahi, M. \& Nawaz, F. IL-12independent Th1-type immune responses to respiratory viral infection: requirement of IL-18 for IFN-gamma release in the lung but not for the differentiation of viral-reactive Th1-type lymphocytes. J. Immunol. 164, 2575-2584 (2000). 